\title{
Conservation of Nucleosome Positions in Duplicated and Orthologous Gene Pairs
}

\author{
Hiromi Nishida \\ Agricultural Bioinformatics Research Unit, Graduate School of Agriculture and Life Sciences, The University of Tokyo, \\ Bunkyo-ku, Tokyo 113-8657, Japan \\ Correspondence should be addressed to Hiromi Nishida, hnishida@iu.a.u-tokyo.ac.jp
}

Received 5 October 2011; Accepted 8 December 2011

Academic Editor: David E. Misek

Copyright ( $\odot 2012$ Hiromi Nishida. This is an open access article distributed under the Creative Commons Attribution License, which permits unrestricted use, distribution, and reproduction in any medium, provided the original work is properly cited.

\begin{abstract}
Although nucleosome positions tend to be conserved in gene promoters, whether they are conserved in duplicated and orthologous genes is unknown. In order to elucidate how nucleosome positions are conserved between duplicated and orthologous gene pairs, I performed 2 comparative studies. First, I compared the nucleosome position profiles of duplicated genes in the filamentous ascomycete Aspergillus fumigatus. After identifying 63 duplicated gene pairs among 9630 protein-encoding genes, I compared the nucleosome position profiles of the paired genes. Although nucleosome positions are conserved more in gene promoters than in gene bodies, their profiles were diverse, suggesting evolutionary changes after gene duplication. Next, I examined the conservation of nucleosome position profiles in $347 \mathrm{~A}$. fumigatus orthologs of S. cerevisiae genes that showed notably high conservation of nucleosome positions between the parent strain and 2 deletion mutants. In only 11 (3.2\%) of the 347 gene pairs, the nucleosome position profile was highly conserved (Spearman's rank correlation coefficient $>0.7$ ). The absence of nucleosome position conservation in promoters of orthologous genes suggests organismal specificity of nucleosome arrangements.
\end{abstract}

\section{Introduction}

Nucleosomes are histone octamers around which DNA is wrapped in 1.65 turns [1]. Neighboring nucleosomes are separated by unwrapped linker DNA. Nucleosome density is lower, and nucleosome position is more conserved in the promoters than in the bodies of genes [2-5]. It is thought that nucleosome positioning in the gene promoter plays an important role in transcriptional regulation.

Although nucleosome positions can be partially simulated using a DNA-sequence-based approach [6], these simulations are limited due to variations between species. The nucleosome positioning mechanism varies between the 2 ascomycetous yeasts, Saccharomyces cerevisiae, and Schizosaccharomyces pombe [7]. Nucleosome positioning differs even among phylogenetically close ascomycetous yeast species [5].

Gene duplication is a driving force behind gene creation, and generating novel functions in newly created genes. Approximately one-half of cellular functions have been gained through gene duplication [8]. The duplicated genes encode similar amino acid sequences and often similar protein functions. It is uncertain, however, whether duplicated genes have similar nucleosome position profiles. In this study, I compared nucleosome positions in the promoter and body regions of duplicated gene pairs in the filamentous ascomycete Aspergillus fumigatus.

Previous analyses have found that nucleosome positions in A. fumigatus are conserved more in gene promoters than in gene bodies, even after treatment with the histone deacetylase inhibitor trichostatin A [4, 9]. In addition, nucleosome positions in S. cerevisiae are more conserved in gene promoters than in gene bodies between the control and the histone acetyltransferase gene ELP3 deletion mutant, and between the control and the histone deacetylase gene HOS2 deletion mutant [10]. The proteins Elp3 and Hos 2 show the highest and the third highest evolutionary conservation, respectively, among the fungal histone modification proteins [11].

How well are nucleosome positions conserved in genes of the same origins? If there is a "nucleosome position code" that regulates nucleosome positioning, common nucleosome 
TABLE 1: Duplicated gene pairs in Aspergillus fumigatus.

\begin{tabular}{|c|c|c|c|c|c|}
\hline \multirow{2}{*}{$\begin{array}{l}\text { Gene pair } \\
\text { AFUA1G00150 }\end{array}$} & \multirow{2}{*}{$\begin{array}{c}\text { Chromosome } \\
1\end{array}$} & \multicolumn{2}{|c|}{ Gene body region } & \multirow{2}{*}{$\begin{array}{c}\text { Gene } \\
\text { direction } \\
+\end{array}$} & \multirow{2}{*}{$\begin{array}{l}\text { Function } \\
\text { RING finger protein }\end{array}$} \\
\hline & & 25442 & 27017 & & \\
\hline AFUA6G09370 & 6 & 2245549 & 2247121 & + & RING finger protein \\
\hline AFUA1G00420 & 1 & 135528 & 137781 & + & Carboxypeptidase S1, putative \\
\hline AFUA8G04120 & 8 & 897824 & 900076 & - & Carboxypeptidase S1, putative \\
\hline AFUA1G00440 & 1 & 138359 & 140093 & - & DUF895 domain membrane protein \\
\hline AFUA8G04110 & 8 & 895512 & 897246 & + & DUF895 domain membrane protein \\
\hline AFUA1G00450 & 1 & 143117 & 144466 & + & $\begin{array}{l}\mathrm{N} \text {-acetylglucosamine-6-phosphate } \\
\text { deacetylase(NagA), putative }\end{array}$ \\
\hline AFUA8G04100 & 8 & 891135 & 892484 & - & $\begin{array}{l}\text { N-acetylglucosamine-6-phosphate } \\
\text { deacetylase(NagA), putative }\end{array}$ \\
\hline AFUA1G00470 & 1 & 148615 & 150219 & + & Betaine aldehyde dehydrogenase, putative \\
\hline AFUA8G04080 & 8 & 885376 & 886980 & - & Betaine aldehyde dehydrogenase (BadH), putative \\
\hline AFUA1G00530 & 1 & 164101 & 164771 & - & Thermoresistant gluconokinase family protein \\
\hline AFUA4G12050 & 4 & 3163747 & 3164530 & - & Thermoresistant gluconokinase \\
\hline AFUA1G00550 & 1 & 177114 & 178593 & + & Hypothetical protein \\
\hline AFUA1G00910 & 1 & 328785 & 330274 & + & Hypothetical protein \\
\hline AFUA1G00580 & 1 & 184790 & 186873 & + & Acid phosphatase (PhoG), putative \\
\hline AFUA8G04050 & 8 & 870757 & 872487 & - & Acid phosphatase (PhoG), putative \\
\hline AFUA1G00650 & 1 & 215584 & 217006 & + & Alpha-1,3-glucanase, putative \\
\hline AFUA7G08510 & 7 & 1973398 & 1974759 & + & Alpha-1,3-glucanase, putative \\
\hline AFUA1G00920 & 1 & 331676 & 332955 & - & Hypothetical protein \\
\hline AFUA3G06425 & 3 & 1582748 & 1584037 & - & Hypothetical protein \\
\hline AFUA1G01050 & 1 & 385302 & 386244 & - & Hypothetical protein \\
\hline AFUA8G06160 & 8 & 1465695 & 1466634 & + & Hypothetical protein \\
\hline AFUA1G02550 & 1 & 744583 & 746335 & - & Tubulin alpha-1 subunit \\
\hline AFUA2G14990 & 2 & 3947008 & 3948834 & - & Tubulin alpha-2 subunit \\
\hline AFUA1G02730 & 1 & 788249 & 789373 & - & $\begin{array}{l}\text { Mitochondrial phosphate carrier protein (Ptp), } \\
\text { putative }\end{array}$ \\
\hline AFUA1G15140 & 1 & 4070230 & 4071449 & - & $\begin{array}{l}\text { Mitochondrial phosphate carrier protein (Mir1), } \\
\text { putative }\end{array}$ \\
\hline AFUA1G05760 & 1 & 1658382 & 1659697 & - & Arsenite efflux transporter \\
\hline AFUA5G15010 & 5 & 3882425 & 3883746 & + & Arsenite permease (ArsB), putative \\
\hline AFUA1G05760 & 1 & 1658382 & 1659697 & - & Arsenite efflux transporter \\
\hline AFUA1G16100 & 1 & 4378898 & 4380216 & + & Arsenite permease (ArsB), putative \\
\hline AFUA1G10910 & 1 & 2848155 & 2850137 & - & Tubulin beta, putative \\
\hline AFUA7G00250 & 7 & 70221 & 71948 & + & Tubulin beta-2 subunit \\
\hline AFUA1G11260 & 1 & 2971529 & 2971889 & - & Conserved hypothetical protein \\
\hline AFUA6G00270 & 6 & 79542 & 79886 & - & Conserved hypothetical protein \\
\hline AFUA1G11610 & 1 & 3060058 & 3060510 & + & 3-Dehydroquinate dehydratase, type II \\
\hline AFUA3G14850 & 3 & 3929721 & 3930173 & + & 3-Dehydroquinate dehydratase, type II \\
\hline AFUA1G11890 & 1 & 3129447 & 3131489 & + & Serine palmitoyltransferase 2 , putative \\
\hline AFUA6G00300 & 6 & 85851 & 87692 & - & Serine palmitoyltransferase 1 , putative \\
\hline AFUA1G12850 & 1 & 3398837 & 3400611 & + & Nitrate transporter (nitrate permease) \\
\hline AFUA1G17470 & 1 & 4782320 & 4783995 & + & High-affinity nitrate transporter NrtB \\
\hline AFUA1G15970 & 1 & 4338407 & 4339712 & + & Aldo-keto reductase (AKR13), putative \\
\hline AFUA8G01560 & 8 & 401581 & 402815 & - & Aldo-keto reductase (YakC), putative \\
\hline AFUA1G16030 & 1 & 4358866 & 4360256 & - & Conserved hypothetical protein \\
\hline AFUA5G14930 & 5 & 3863218 & 3864521 & - & Conserved hypothetical protein \\
\hline
\end{tabular}


Table 1: Continued.

\begin{tabular}{|c|c|c|c|c|c|}
\hline \multirow{2}{*}{$\begin{array}{l}\text { Gene pair } \\
\text { AFUA1G16040 }\end{array}$} & \multirow{2}{*}{$\begin{array}{c}\text { Chromosome } \\
1\end{array}$} & \multicolumn{2}{|c|}{ Gene body region } & \multirow{2}{*}{$\begin{array}{c}\text { Gene } \\
\text { direction } \\
+\end{array}$} & \multirow{2}{*}{$\begin{array}{l}\text { Function } \\
\text { Metalloreductase, putative }\end{array}$} \\
\hline & & 4363493 & 4365310 & & \\
\hline AFUA5G14940 & 5 & 3867797 & 3869542 & + & Cell surface metalloreductase (FreA), putative \\
\hline AFUA1G16050 & 1 & 4366573 & 4368232 & + & Hypothetical protein \\
\hline AFUA5G14950 & 5 & 3870635 & 3872465 & + & Hypothetical protein \\
\hline AFUA1G16070 & 1 & 4370346 & 4373694 & + & Conserved hypothetical protein \\
\hline AFUA5G14980 & 5 & 3874539 & 3877890 & + & Conserved hypothetical protein \\
\hline AFUA1G16080 & 1 & 4374579 & 4375298 & - & Hypothetical protein \\
\hline AFUA5G14990 & 5 & 3878797 & 3879579 & - & Hypothetical protein \\
\hline AFUA1G16090 & 1 & 4377842 & 4378249 & - & Arsenate reductase (ArsC), putative \\
\hline AFUA5G15000 & 5 & 3881386 & 3881835 & - & Arsenate reductase (ArsC), putative \\
\hline AFUA1G16100 & 1 & 4378898 & 4380216 & + & Arsenite permease (ArsB), putative \\
\hline AFUA5G15010 & 5 & 3882425 & 3883746 & + & Arsenite permease (ArsB), putative \\
\hline AFUA1G16110 & 1 & 4380474 & 4381455 & - & Arsenic methyltransferase (Cyt19), putative \\
\hline AFUA5G15020 & 5 & 3883929 & 3884993 & - & Arsenic methyltransferase (Cyt19), putative \\
\hline AFUA1G16120 & 1 & 4385650 & 4386671 & - & Arsenic resistance protein $(\mathrm{ArsH})$, putative \\
\hline AFUA8G07150 & 8 & 1751693 & 1752688 & + & ArsH protein \\
\hline AFUA1G16120 & 1 & 4385650 & 4386671 & - & Arsenic resistance protein $(\mathrm{ArsH})$, putative \\
\hline AFUA5G15030 & 5 & 3887287 & 3888230 & - & Arsenic resistance protein $(\mathrm{ArsH})$, putative \\
\hline AFUA2G00800 & 2 & 178081 & 179459 & - & PelA protein \\
\hline AFUA5G10380 & 5 & 2658656 & 2659968 & + & Pectin lyase, putative \\
\hline AFUA2G00800 & 2 & 178081 & 179459 & - & PelA protein \\
\hline AFUA7G05030 & 7 & 1182104 & 1183794 & - & Pectin lyase B \\
\hline AFUA2G04010 & 2 & 1092973 & 1094679 & - & $\begin{array}{l}\text { Alpha, alpha-trehalose-phosphate synthase } \\
\text { subunit, putative }\end{array}$ \\
\hline AFUA6G12950 & 6 & 3268958 & 3270783 & + & $\begin{array}{l}\text { Alpha, alpha-trehalose-phosphate Synthase } \\
\text { subunitTPS1, putative }\end{array}$ \\
\hline AFUA2G11270 & 2 & 2897275 & 2904954 & - & Alpha-1,3-glucan synthase, putative \\
\hline AFUA3G00910 & 3 & 210186 & 217666 & + & Alpha-1,3-glucan synthase, putative \\
\hline AFUA3G00340 & 3 & 71186 & 72734 & + & Glycosyl hydrolase, putative \\
\hline AFUA4G02720 & 4 & 751306 & 752715 & - & Glycosyl hydrolase, putative \\
\hline AFUA3G00680 & 3 & 151397 & 153549 & - & Copper amine oxidase \\
\hline AFUA7G04180 & 7 & 943893 & 946136 & + & Amine oxidase \\
\hline AFUA3G01560 & 3 & 393032 & 394802 & - & Aminoacid permease, putative \\
\hline AFUA5G04260 & 5 & 1140953 & 1142714 & + & Arginine transporter, putative \\
\hline AFUA3G02420 & 3 & 597476 & 598286 & + & ThiJ/PfpI family protein \\
\hline AFUA4G01400 & 4 & 369123 & 369944 & - & ThiJ/PfpI family protein \\
\hline AFUA3G03080 & 3 & 824479 & 825425 & + & Endo-1,3(4)-beta-glucanase, putative \\
\hline AFUA6G14540 & 6 & 3702416 & 3703383 & - & Endo-1,3(4)-beta-glucanase, putative \\
\hline AFUA3G03980 & 3 & 1134609 & 1136404 & + & Cytochrome P450 monooxygenase, putative \\
\hline AFUA5G10050 & 5 & 2589301 & 2591081 & - & Cytochrome P450 monooxygenase, putative \\
\hline AFUA3G08160 & 3 & 2094223 & 2095759 & - & $\begin{array}{l}\text { Eukaryotic translation initiation Factor eIF4A, } \\
\text { putative }\end{array}$ \\
\hline AFUA5G02410 & 5 & 621886 & 623486 & - & DEAD/DEAH box helicase, putative \\
\hline AFUA3G14420 & 3 & 3831868 & 3834921 & + & Chitin synthase G \\
\hline AFUA5G00760 & 5 & 211013 & 213795 & + & Chitin synthase C \\
\hline AFUA4G00510 & 4 & 133477 & 135102 & - & Hypothetical protein \\
\hline AFUA7G08600 & 7 & 2009436 & 2011052 & + & Hypothetical protein \\
\hline AFUA4G03110 & 4 & 868474 & 870249 & - & Monosaccharide transporter \\
\hline AFUA5G10690 & 5 & 2737348 & 2739178 & - & Monosaccharide transporter \\
\hline
\end{tabular}


TABle 1: Continued.

\begin{tabular}{|c|c|c|c|c|c|}
\hline Gene pair & Chromosome & \multicolumn{2}{|c|}{ Gene body region } & \multirow{2}{*}{$\begin{array}{c}\text { Gene } \\
\text { direction } \\
-\end{array}$} & \multirow{2}{*}{$\begin{array}{l}\text { Function } \\
\text { Oxidoreductase, short-chain } \\
\text { dehydrogenase/reductase family }\end{array}$} \\
\hline AFUA4G03680 & 4 & 1031586 & 1032539 & & \\
\hline AFUA6G03520 & 6 & 764308 & 765294 & - & $\begin{array}{l}\text { Short-chain dehydrogenase/reductase family } \\
\text { protein, putative }\end{array}$ \\
\hline AFUA4G09440 & 4 & 2462920 & 2466158 & - & Sodium P-type ATPase, putative \\
\hline AFUA6G03690 & 6 & 810027 & 813362 & - & Sodium transport ATPase, putative \\
\hline AFUA4G14360 & 4 & 3774307 & 3776166 & + & Capsular associated protein, putative \\
\hline AFUA5G07560 & 5 & 1889791 & 1891689 & - & Capsular associated protein, putative \\
\hline AFUA5G00145 & 5 & 15749 & 16327 & - & Hypothetical protein \\
\hline AFUA6G11710 & 6 & 2915903 & 2916483 & + & Conserved hypothetical protein \\
\hline AFUA5G00145 & 5 & 15749 & 16327 & - & Hypothetical protein \\
\hline AFUA7G08440 & 7 & 1942319 & 1942804 & + & Hypothetical protein \\
\hline AFUA5G01030 & 5 & 266294 & 267439 & - & $\begin{array}{l}\text { Glyceraldehyde 3-phosphate } \\
\text { dehydrogenase(Ccg-7), putative }\end{array}$ \\
\hline AFUA5G01970 & 5 & 503797 & 505194 & + & $\begin{array}{l}\text { Glyceraldehyde 3-phosphate dehydrogenase } \\
\text { GpdA }\end{array}$ \\
\hline AFUA5G06240 & 5 & 1494455 & 1495619 & - & Alcohol dehydrogenase, putative \\
\hline AFUA7G01010 & 7 & 270494 & 271675 & - & Alcohol dehydrogenase, putative \\
\hline AFUA5G07980 & 5 & 2019069 & 2020841 & + & Hypothetical protein \\
\hline AFUA5G14920 & 5 & 3857350 & 3859167 & + & Hypothetical protein \\
\hline AFUA5G09130 & 5 & 2345689 & 2346728 & - & Polysaccharide deacetylase family protein \\
\hline AFUA6G05030 & 6 & 1195846 & 1196956 & + & Polysaccharide deacetylase family protein \\
\hline AFUA5G15030 & 5 & 3887287 & 3888230 & - & Arsenic resistance protein $(\mathrm{ArsH})$, putative \\
\hline AFUA8G07150 & 8 & 1751693 & 1752688 & + & ArsH protein \\
\hline AFUA6G06750 & 6 & 1475239 & 1476209 & + & 14-3-3 family protein \\
\hline AFUA2G03290 & 2 & 867203 & 868250 & - & 14-3-3 family protein ArtA, putative \\
\hline AFUA6G07070 & 6 & 1587643 & 1589124 & + & Cellobiohydrolase D \\
\hline AFUA6G11610 & 6 & 2878078 & 2879676 & - & 1,4-beta-D-glucan-cellobiohydrolyase, putative \\
\hline AFUA6G11430 & 6 & 2837140 & 2839051 & + & Aldehyde dehydrogenase, putative \\
\hline AFUA7G01000 & 7 & 267518 & 269163 & - & Aldehyde dehydrogenase, putative \\
\hline AFUA6G11710 & 6 & 2915903 & 2916483 & + & Conserved hypothetical protein \\
\hline AFUA7G08440 & 7 & 1942319 & 1942804 & + & Hypothetical protein \\
\hline AFUA6G13490 & 6 & 3431912 & 3433612 & - & Glutamate decarboxylase \\
\hline AFUA8G06020 & 8 & 1428812 & 1430515 & + & Glutamate decarboxylase \\
\hline AFUA7G00360 & 7 & 102030 & 103064 & - & UDP-galactose 4-epimerase, putative \\
\hline AFUA8G00860 & 8 & 203496 & 204338 & + & UDP-galactose 4-epimerase, putative \\
\hline AFUA7G07050 & 7 & 1725686 & 1726402 & - & Hypothetical protein \\
\hline AFUA7G08300 & 7 & 1870244 & 1870960 & - & Hypothetical protein \\
\hline AFUA7G07060 & 7 & 1728876 & 1732992 & - & Hypothetical protein \\
\hline AFUA7G08310 & 7 & 1873437 & 1877817 & - & Hypothetical protein \\
\hline
\end{tabular}

positions should remain in the promoters of orthologous genes across distinct species. In this study, I compared nucleosome positions in the promoters of duplicated and orthologous genes in A. fumigatus and S. cerevisiae.

\section{Materials and Methods}

2.1. Identification of Duplicated Gene Pairs in Aspergillus fumigatus. Protein-coding gene pairs aligned over more than $80 \%$ of query length and more than $70 \%$ aminoacid sequence identity were selected by performing a BLAST search of 9630 A. fumigatus proteins at Fungal Genomes Central on NCBI (http://www.ncbi.nlm.nih.gov/projects/genome/guide/fungi/). Pairs in which the lengths differ by more than $25 \%$ were not used. Thus, we identified 63 duplicated A. fumigatus gene pairs (Tables 1 and 2).

2.2. Identification of Orthologous Gene Pairs in Aspergillus fumigatus and Saccharomyces cerevisiae. In a comparison 
TABLE 2: Spearman's rank correlation coefficients of nucleosome position profiles in the promoter and body regions of 63 duplicated gene pairs in Aspergillus fumigatus.

\begin{tabular}{|c|c|c|c|}
\hline \multicolumn{2}{|c|}{ Gene pair } & \multirow{2}{*}{$\begin{array}{c}\text { Gene promoter } \\
0.750176913\end{array}$} & \multirow{2}{*}{$\begin{array}{c}\text { Gene body } \\
0.215204254\end{array}$} \\
\hline AFUA1G00150 & AFUA6G09370 & & \\
\hline AFUA1G00420 & AFUA8G04120 & 0.80370974 & 0.723149072 \\
\hline AFUA1G00440 & AFUA8G04110 & 0.856048561 & 0.225149091 \\
\hline AFUA1G00450 & AFUA8G04100 & 0.9213602 & 0.94633912 \\
\hline AFUA1G00470 & AFUA8G04080 & 0.433118045 & 0.818800395 \\
\hline AFUA1G00530 & AFUA4G12050 & 0.326735006 & -0.199291887 \\
\hline AFUA1G00550 & AFUA1G00910 & 0.676910334 & 0.678763937 \\
\hline AFUA1G00580 & AFUA8G04050 & 0.305291793 & 0.312927993 \\
\hline AFUA1G00650 & AFUA7G08510 & 0.448171525 & 0.085599209 \\
\hline AFUA1G00920 & AFUA3G06425 & 0.719748778 & -0.340001527 \\
\hline AFUA1G01050 & AFUA8G06160 & 0.635149308 & 0.176679577 \\
\hline AFUA1G02550 & AFUA2G14990 & 0.139079116 & -0.036644732 \\
\hline AFUA1G02730 & AFUA1G15140 & 0.452193259 & 0.067186644 \\
\hline AFUA1G05760 & AFUA5G15010 & 0.395849711 & 0.117006666 \\
\hline AFUA1G05760 & AFUA1G16100 & 0.127911436 & -0.397363108 \\
\hline AFUA1G10910 & AFUA7G00250 & 0.310718577 & 0.665906466 \\
\hline AFUA1G11260 & AFUA6G00270 & 0.617303556 & -0.02397179 \\
\hline AFUA1G11610 & AFUA3G14850 & 0.636281069 & 0.869300724 \\
\hline AFUA1G11890 & AFUA6G00300 & 0.254487564 & 0.429087849 \\
\hline AFUA1G12850 & AFUA1G17470 & 0.644447287 & 0.008468365 \\
\hline AFUA1G15970 & AFUA8G01560 & 0.373850536 & -0.106800689 \\
\hline AFUA1G16030 & AFUA5G14930 & 0.618349765 & 0.858488283 \\
\hline AFUA1G16040 & AFUA5G14940 & 0.481054005 & 0.017330056 \\
\hline AFUA1G16050 & AFUA5G14950 & -0.345037268 & -0.392132138 \\
\hline AFUA1G16070 & AFUA5G14980 & 0.930922124 & 0.902581516 \\
\hline AFUA1G16080 & AFUA5G14990 & 0.559980787 & 0.136819379 \\
\hline AFUA1G16090 & AFUA5G15000 & 0.773867924 & 0.338119153 \\
\hline AFUA1G16100 & AFUA5G15010 & 0.681170098 & 0.70434823 \\
\hline AFUA1G16110 & AFUA5G15020 & -0.261864905 & 0.632982505 \\
\hline AFUA1G16120 & AFUA8G07150 & 0.356869915 & 0.074502754 \\
\hline AFUA1G16120 & AFUA5G15030 & -0.135442129 & -0.239074416 \\
\hline AFUA2G00800 & AFUA5G10380 & 0.352063916 & 0.460712971 \\
\hline AFUA2G00800 & AFUA7G05030 & -0.114184443 & 0.457081245 \\
\hline AFUA2G04010 & AFUA6G12950 & 0.134790545 & -0.14813806 \\
\hline AFUA2G11270 & AFUA3G00910 & -0.004207858 & 0.192024608 \\
\hline AFUA3G00340 & AFUA4G02720 & -0.050259987 & 0.055757479 \\
\hline AFUA3G00680 & AFUA7G04180 & 0.399912713 & -0.044615588 \\
\hline AFUA3G01560 & AFUA5G04260 & 0.179395067 & 0.125177632 \\
\hline AFUA3G02420 & AFUA4G01400 & 0.662712481 & 0.646400554 \\
\hline AFUA3G03080 & AFUA6G14540 & 0.3401707 & -0.021961056 \\
\hline AFUA3G03980 & AFUA5G10050 & 0.486726534 & 0.318475376 \\
\hline AFUA3G08160 & AFUA5G02410 & 0.309645464 & -0.255654021 \\
\hline AFUA3G14420 & AFUA5G00760 & 0.917685134 & 0.415111431 \\
\hline AFUA4G00510 & AFUA7G08600 & 0.578582743 & 0.441363777 \\
\hline AFUA4G03110 & AFUA5G10690 & 0.721540193 & 0.176996881 \\
\hline AFUA4G03680 & AFUA6G03520 & 0.662657833 & -0.320417166 \\
\hline AFUA4G09440 & AFUA6G03690 & 0.204159184 & 0.000609208 \\
\hline AFUA4G14360 & AFUA5G07560 & 0.038083073 & 0.515103795 \\
\hline AFUA5G00145 & AFUA6G11710 & 0.809402393 & -0.741045496 \\
\hline
\end{tabular}


TABle 2: Continued.

\begin{tabular}{|c|c|c|c|}
\hline \multicolumn{2}{|c|}{ Gene pair } & Gene promoter & Gene body \\
\hline AFUA5G00145 & AFUA7G08440 & 0.019652845 & -0.575600966 \\
\hline AFUA5G01030 & AFUA5G01970 & 0.576211178 & 0.132479521 \\
\hline AFUA5G06240 & AFUA7G01010 & 0.163674862 & -0.552899419 \\
\hline AFUA5G07980 & AFUA5G14920 & 0.669562689 & -0.265207346 \\
\hline AFUA5G09130 & AFUA6G05030 & 0.092452938 & 0.729278817 \\
\hline AFUA5G15030 & AFUA8G07150 & 0.337482368 & -0.502391454 \\
\hline AFUA6G06750 & AFUA2G03290 & 0.333402926 & -0.538389013 \\
\hline AFUA6G07070 & AFUA6G11610 & -0.048961745 & -0.239430043 \\
\hline AFUA6G11430 & AFUA7G01000 & 0.0145492 & 0.476692417 \\
\hline AFUA6G11710 & AFUA7G08440 & 0.243362623 & 0.823124582 \\
\hline AFUA6G13490 & AFUA8G06020 & 0.768186533 & 0.174847009 \\
\hline AFUA7G00360 & AFUA8G00860 & -0.115050268 & 0.03815086 \\
\hline AFUA7G07050 & AFUA7G08300 & 0.70756842 & 0.778653567 \\
\hline AFUA7G07060 & AFUA7G08310 & 0.906852725 & 0.919839072 \\
\hline
\end{tabular}

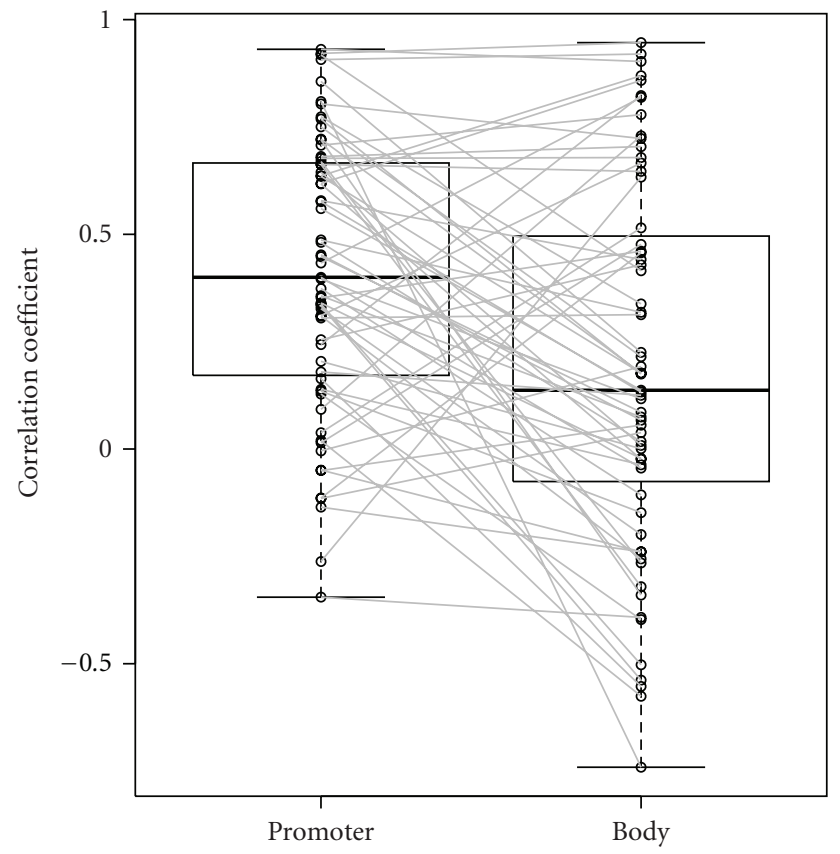

FIGURE 1: Boxplots of Spearman's rank correlation coefficients of nucleosome position profiles in the promoter and body regions of 63 duplicated gene pairs. Circles represent the correlation coefficients and values of the same genes are connected by lines.

of nucleosome positioning between $A$. fumigatus and $S$. cerevisiae, I focused on 466 genes (Table 3) that showed notably high conservation of nucleosome positioning in the promoters of the control and the ELP3 and HOS2 deletion mutants from the previous study [10].

A total of 3339 ortholog clusters were identified (See table 1 in Supplementary Material available at doi: 10.1100/2012/298174) between A. fumigatus and S. cerevisiae by ortholog cluster analysis in the Microbial Genome Database for Comparative Analysis (MBGD, http://mbgd .nibb.ac.jp/) [12]. Of these orthologous gene pairs, 347 (Table 4) are yeast genes that showed a high level of

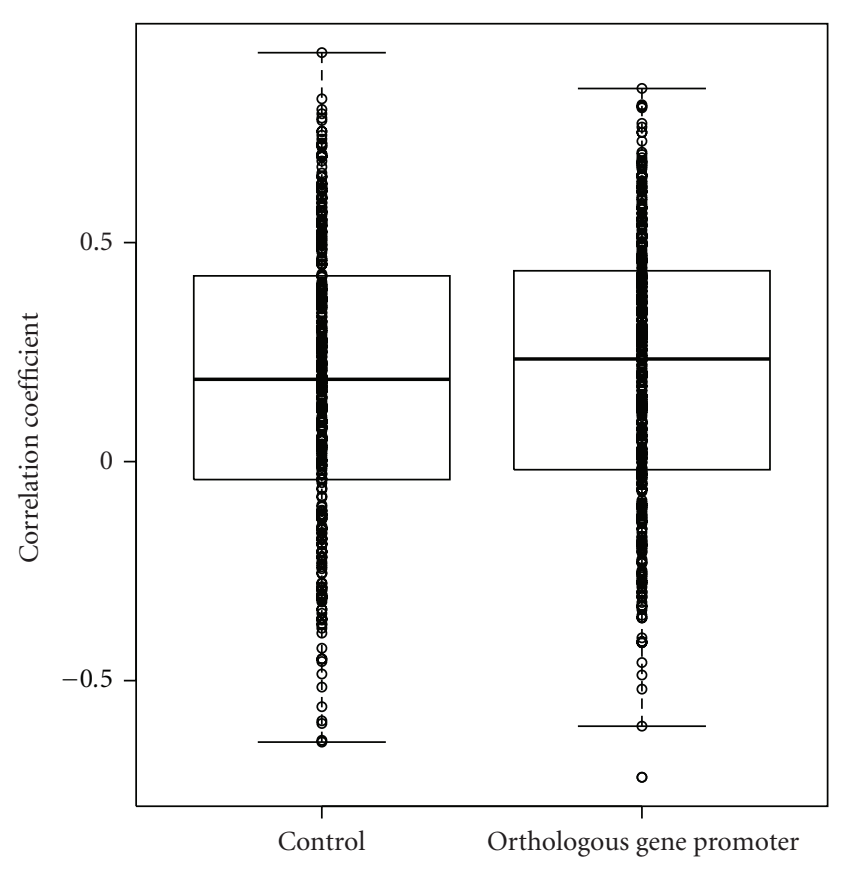

FIGURE 2: Boxplots of Spearman's rank correlation coefficients between nucleosome position profiles in the promoters of 347 orthologous gene pairs between Aspergillus fumigatus and Saccharomyces cerevisiae. The same number of gene pairs was chosen at random to serve as a control. Dots indicate correlation coefficients. The distributions of correlation coefficients did not significantly differ $(P$-value $=0.28$ in Kolmogorov-Smirnov test $)$ between the orthologous gene promoters and the controls.

nucleosome positioning conservation in the control and deletion mutants. I focused on these 347 orthologous pairs to compare nucleosome positioning between species. The same number of pairs of $A$. fumigatus and $S$. cerevisiae genes chosen at random were used as a control.

2.3. Nucleosome Position Profile. Nucleosome mapping numbers at each genomic position were determined [13] based 
Table 3: Genes of Saccharomyces cerevisiae with highly conserved nucleosome positions in the promoters of the control and histone modification gene deletion mutants.

\begin{tabular}{|c|c|c|c|c|c|}
\hline Chromosome & Gene & $\begin{array}{l}\text { Correlation } \\
\text { coefficient between } \\
\text { the control and the } \\
\text { ELP3 deletion }\end{array}$ & $\begin{array}{l}\text { Correlation } \\
\text { coefficient between } \\
\text { the control and the } \\
\text { HOS } 2 \text { deletion }\end{array}$ & $\begin{array}{l}\text { Translational } \\
\text { start site }\end{array}$ & $\begin{array}{c}\text { Transcription } \\
\text { direction }\end{array}$ \\
\hline chr01 & YAL064W-B & 0.974719955 & 0.965814243 & 12047 & + \\
\hline chr01 & YAL056W & 0.989801003 & 0.982157328 & 39260 & + \\
\hline chr01 & YAL047C & 0.950243691 & 0.953129232 & 56858 & - \\
\hline chr01 & YAR019C & 0.970115162 & 0.960628643 & 175133 & - \\
\hline chr01 & YAR033W & 0.968807875 & 0.987221166 & 188101 & + \\
\hline chr02 & YBL111C & 0.995802065 & 0.99455065 & 5009 & - \\
\hline chr02 & $Y B L 108 C-A$ & 0.959497013 & 0.969966542 & 7733 & - \\
\hline chr02 & YBL101C & 0.958517125 & 0.991324096 & 28299 & - \\
\hline chr02 & YBL087C & 0.95719187 & 0.957273203 & 60735 & - \\
\hline chr02 & YBL061C & 0.984960212 & 0.992728731 & 107408 & - \\
\hline chr02 & YBL060W & 0.960330289 & 0.99357258 & 107934 & + \\
\hline chr02 & YBL051C & 0.97653734 & 0.989165943 & 124762 & - \\
\hline chr02 & $Y B L 032 W$ & 0.956874854 & 0.980366627 & 160187 & + \\
\hline chr02 & $Y B L 005 W-B$ & 0.950781322 & 0.952679326 & 221333 & + \\
\hline chr02 & $Y B L 005 W-A$ & 0.950781322 & 0.952679326 & 221333 & + \\
\hline chr02 & YBR023C & 0.960238499 & 0.992906362 & 287925 & - \\
\hline chr02 & YBR029C & 0.959231389 & 0.987222377 & 297742 & - \\
\hline chr02 & YBR047W & 0.964564522 & 0.963289004 & 331831 & + \\
\hline chr02 & YBR060C & 0.959229871 & 0.974718825 & 362512 & - \\
\hline chr02 & YBR084W & 0.962172866 & 0.981530879 & 411048 & + \\
\hline chr02 & YBR090C & 0.967658785 & 0.971792497 & 427052 & - \\
\hline chr02 & YBR091C & 0.965037862 & 0.985960321 & 427478 & - \\
\hline chr02 & YBR $131 W$ & 0.953323249 & 0.969685452 & 497157 & + \\
\hline chr02 & YBR136W & 0.963546822 & 0.974854426 & 505662 & + \\
\hline chr02 & YBR173C & 0.978518391 & 0.961438459 & 582167 & - \\
\hline chr02 & YBR179C & 0.988489146 & 0.991754907 & 589109 & - \\
\hline chr02 & YBR180W & 0.987132212 & 0.975974209 & 589736 & + \\
\hline chr02 & YBR204C & 0.970958946 & 0.978433534 & 633376 & - \\
\hline chr02 & $Y B R 243 C$ & 0.972253467 & 0.961252065 & 706788 & - \\
\hline chr02 & YBR244W & 0.974202306 & 0.992702483 & 707523 & + \\
\hline chr02 & YBR249C & 0.955742682 & 0.976101912 & 717989 & - \\
\hline chr02 & YBR250W & 0.954149188 & 0.976352348 & 719028 & + \\
\hline chr02 & YBR251W & 0.96273261 & 0.961120273 & 721385 & + \\
\hline chr02 & YBR258C & 0.966601899 & 0.955155711 & 730157 & - \\
\hline chr02 & YBR260C & 0.969346019 & 0.970918059 & 734634 & - \\
\hline chr02 & YBR279W & 0.956081387 & 0.964626558 & 761253 & + \\
\hline chr02 & YBR290W & 0.981586823 & 0.967935658 & 782587 & + \\
\hline chr03 & YCL064C & 0.957150698 & 0.95270514 & 16880 & - \\
\hline chr03 & YCL058W-A & 0.969895345 & 0.992394829 & 23584 & + \\
\hline chr03 & YCL057C-A & 0.990886192 & 0.987170576 & 24325 & - \\
\hline chr03 & YCL057W & 0.963062357 & 0.961804623 & 24768 & + \\
\hline chr03 & YCLO38C & 0.978210537 & 0.957633548 & 56527 & - \\
\hline chr03 & YCLO35C & 0.981360705 & 0.973568151 & 61173 & - \\
\hline chr03 & YCL019W & 0.953241664 & 0.978688459 & 85102 & + \\
\hline chr03 & YCR026C & 0.96886665 & 0.97932414 & 166335 & - \\
\hline chr03 & YCR043C & 0.951764864 & 0.966166673 & 206640 & - \\
\hline
\end{tabular}


Table 3: Continued.

\begin{tabular}{|c|c|c|c|c|c|}
\hline Chromosome & Gene & $\begin{array}{l}\text { Correlation } \\
\text { coefficient between } \\
\text { the control and the } \\
\text { ELP3 deletion }\end{array}$ & $\begin{array}{l}\text { Correlation } \\
\text { coefficient between } \\
\text { the control and the } \\
\text { HOS } 2 \text { deletion }\end{array}$ & $\begin{array}{l}\text { Translational } \\
\text { start site }\end{array}$ & $\begin{array}{c}\text { Transcription } \\
\text { direction }\end{array}$ \\
\hline chr03 & YCR053W & 0.989728241 & 0.992623598 & 216693 & + \\
\hline chr03 & YCR087C-A & 0.975398919 & 0.993232435 & 264464 & - \\
\hline chr03 & YCR090C & 0.965637294 & 0.95018878 & 272860 & - \\
\hline chr03 & YCR108C & 0.985803086 & 0.993860549 & 316185 & - \\
\hline chr04 & YDL248W & 0.964701365 & 0.980217326 & 1802 & + \\
\hline chr04 & YDL247W & 0.989686199 & 0.997997741 & 5985 & + \\
\hline chr04 & YDL233W & 0.957672252 & 0.97857489 & 36798 & + \\
\hline chr04 & $Y D L 232 W$ & 0.979592686 & 0.970413477 & 38488 & + \\
\hline chr04 & YDL225W & 0.950053133 & 0.978441348 & 52446 & + \\
\hline chr04 & YDL208W & 0.987264893 & 0.987894794 & 87513 & + \\
\hline chr04 & YDL189W & 0.965815356 & 0.98561241 & 122217 & + \\
\hline chr04 & $Y D L 174 C$ & 0.956215571 & 0.953787348 & 147590 & - \\
\hline chr04 & $Y D L 147 W$ & 0.971755059 & 0.950911485 & 190925 & + \\
\hline chr04 & YDL116W & 0.965936123 & 0.976379579 & 251566 & + \\
\hline chr04 & $Y D L 110 C$ & 0.974238254 & 0.97295169 & 264964 & - \\
\hline chr04 & YDL102W & 0.957786538 & 0.980122821 & 276872 & + \\
\hline chr04 & YDL085W & 0.97887937 & 0.960150217 & 303211 & + \\
\hline chr04 & $Y D L 035 C$ & 0.975573212 & 0.977145228 & 392054 & - \\
\hline chr04 & YDL025C & 0.96134673 & 0.972526893 & 407203 & - \\
\hline chr04 & YDR019C & 0.95269772 & 0.953635877 & 485362 & - \\
\hline chr04 & YDR028C & 0.960937436 & 0.959981321 & 500876 & - \\
\hline chr04 & YDR034C-C & 0.967222636 & 0.9814374 & 519353 & - \\
\hline chr04 & YDR034C-D & 0.967222636 & 0.981437429 & 519353 & - \\
\hline chr04 & YDR037W & 0.951365292 & 0.956861294 & 525437 & + \\
\hline chr04 & YDR054C & 0.955928802 & 0.982048103 & 562325 & - \\
\hline chr04 & YDR055W & 0.950321012 & 0.952540035 & 563525 & + \\
\hline chr04 & YDR062W & 0.974021833 & 0.967688258 & 576471 & + \\
\hline chr04 & YDR109C & 0.954396163 & 0.952291074 & 675664 & - \\
\hline chr04 & YDR110W & 0.986081905 & 0.974555387 & 676099 & + \\
\hline chr04 & YDR120C & 0.960200845 & 0.969981962 & 693258 & - \\
\hline chr04 & YDR162C & 0.967301741 & 0.984347121 & 781097 & - \\
\hline chr04 & YDR233C & 0.967024058 & 0.967951444 & 930353 & - \\
\hline chr04 & $Y D R 234 W$ & 0.967415159 & 0.980945022 & 931125 & + \\
\hline chr04 & $Y D R 238 C$ & 0.97602642 & 0.973622232 & 940812 & - \\
\hline chr04 & $Y D R 261 C-D$ & 0.984848646 & 0.993722663 & 992345 & - \\
\hline chr04 & YDR261C-C & 0.984848646 & 0.9937227 & 992345 & - \\
\hline chr04 & $Y D R 262 W$ & 0.990989397 & 0.995915841 & 993130 & + \\
\hline chr04 & YDR270W & 0.951139826 & 0.96263534 & 1005671 & + \\
\hline chr04 & $Y D R 281 C$ & 0.984619329 & 0.959392909 & 1022317 & - \\
\hline chr04 & YDR300C & 0.975849644 & 0.971677938 & 1062787 & - \\
\hline chr04 & YDR301W & 0.952923341 & 0.977515222 & 1063348 & + \\
\hline chr04 & YDR307W & 0.951918886 & 0.970033626 & 1075861 & + \\
\hline chr04 & YDR310C & 0.951723931 & 0.969273153 & 1084312 & - \\
\hline chr04 & YDR311W & 0.953685357 & 0.980595738 & 1085062 & + \\
\hline chr04 & YDR317W & 0.98969221 & 0.991598049 & 1102181 & + \\
\hline chr04 & YDR322W & 0.954843272 & 0.97204312 & 1110586 & + \\
\hline chr04 & YDR328C & 0.957421993 & 0.983957835 & 1126013 & - \\
\hline chr04 & YDR334W & 0.953030288 & 0.963155512 & 1135927 & + \\
\hline
\end{tabular}


Table 3: Continued.

\begin{tabular}{|c|c|c|c|c|c|}
\hline Chromosome & Gene & $\begin{array}{l}\text { Correlation } \\
\text { coefficient between } \\
\text { the control and the } \\
\text { ELP3 deletion }\end{array}$ & $\begin{array}{l}\text { Correlation } \\
\text { coefficient between } \\
\text { the control and the } \\
\text { HOS } 2 \text { deletion }\end{array}$ & $\begin{array}{l}\text { Translational } \\
\text { start site }\end{array}$ & $\begin{array}{c}\text { Transcription } \\
\text { direction }\end{array}$ \\
\hline chr04 & YDR359C & 0.977489172 & 0.969606029 & 1194877 & - \\
\hline chr04 & YDR365C & 0.985073544 & 0.998926611 & 1206375 & - \\
\hline chr04 & YDR367W & 0.990041805 & 0.994689201 & 1212840 & + \\
\hline chr04 & $Y D R 369 C$ & 0.957655919 & 0.96987305 & 1217572 & - \\
\hline chr04 & YDR379W & 0.962899057 & 0.974939821 & 1230159 & + \\
\hline chr04 & YDR397C & 0.966298918 & 0.975133337 & 1266890 & - \\
\hline chr04 & YDR420W & 0.971217177 & 0.991886941 & 1306259 & + \\
\hline chr04 & YDR424C & 0.966881808 & 0.989813233 & 1319833 & - \\
\hline chr04 & YDR432W & 0.955728738 & 0.982171718 & 1328775 & + \\
\hline chr04 & YDR438W & 0.971626925 & 0.98904082 & 1338266 & + \\
\hline chr04 & $Y D R 444 W$ & 0.973566433 & 0.976502447 & 1350282 & + \\
\hline chr04 & YDR453C & 0.952661492 & 0.954481014 & 1365654 & - \\
\hline chr04 & YDR476C & 0.969940159 & 0.964679279 & 1411119 & - \\
\hline chr04 & YDR477W & 0.954743343 & 0.979243738 & 1412365 & + \\
\hline chr04 & YDR479C & 0.977460825 & 0.994501786 & 1416866 & - \\
\hline chr04 & YDR480W & 0.967727074 & 0.986953403 & 1417391 & + \\
\hline chr04 & $Y D R 488 C$ & 0.983114512 & 0.979187155 & 1430781 & - \\
\hline chr04 & YDR497C & 0.955855412 & 0.958236577 & 1445459 & - \\
\hline chr04 & YDR529C & 0.966273576 & 0.971812076 & 1496540 & - \\
\hline chr05 & YEL072W & 0.968131913 & 0.977435534 & 13720 & + \\
\hline chr05 & YEL043W & 0.979079858 & 0.96227706 & 70478 & + \\
\hline chr05 & YEL038W & 0.981572221 & 0.988369151 & 80462 & + \\
\hline chr05 & YEL021W & 0.951315475 & 0.951565922 & 116167 & + \\
\hline chr05 & YER004W & 0.956348638 & 0.987671859 & 159579 & + \\
\hline chr05 & YER026C & 0.964952897 & 0.993875293 & 208473 & - \\
\hline chr05 & YER076C & 0.965398244 & 0.971982059 & 313494 & - \\
\hline chr05 & YER083C & 0.953185048 & 0.981521771 & 327027 & - \\
\hline chr05 & YER094C & 0.976027049 & 0.982595427 & 349342 & - \\
\hline chr05 & YER095W & 0.963423619 & 0.988752501 & 349976 & + \\
\hline chr05 & YER107C & 0.982024941 & 0.98488468 & 374541 & - \\
\hline chr05 & YER109C & 0.969371754 & 0.986034001 & 377610 & - \\
\hline chr05 & YER173W & 0.960496827 & 0.952930419 & 536295 & + \\
\hline chr05 & YER188C-A & 0.991489293 & 0.996538184 & 569902 & - \\
\hline chr05 & YER189W & 0.997057108 & 0.998333831 & 571150 & + \\
\hline chr06 & YFL066C & 0.970012372 & 0.993098415 & 2615 & - \\
\hline chr06 & YFL065C & 0.957034943 & 0.97004682 & 3338 & - \\
\hline chr06 & YFL060C & 0.987757312 & 0.995138274 & 10969 & - \\
\hline chr06 & YFL059W & 0.976205235 & 0.962195117 & 11363 & + \\
\hline chr06 & YFL058W & 0.9750941 & 0.994474768 & 12929 & + \\
\hline chr06 & YFL028C & 0.966244522 & 0.959308346 & 80211 & - \\
\hline chr06 & YFL026W & 0.957755207 & 0.952711873 & 82578 & + \\
\hline chr06 & YFR009W & 0.979200258 & 0.985708335 & 162482 & + \\
\hline chr06 & YFR013W & 0.972958971 & 0.986214291 & 169914 & + \\
\hline chr06 & YFR037C & 0.965415915 & 0.972070667 & 229173 & - \\
\hline chr07 & YGL255W & 0.961366208 & 0.972062316 & 20978 & + \\
\hline chr07 & YGL248W & 0.971369747 & 0.971756841 & 35653 & + \\
\hline chr07 & YGL223C & 0.956215218 & 0.984994924 & 80364 & - \\
\hline chr07 & YGL215W & 0.965861339 & 0.95181154 & 87980 & + \\
\hline chr07 & YGL201C & 0.954296336 & 0.979120204 & 120911 & - \\
\hline
\end{tabular}


Table 3: Continued.

\begin{tabular}{|c|c|c|c|c|c|}
\hline Chromosome & Gene & $\begin{array}{l}\text { Correlation } \\
\text { coefficient between } \\
\text { the control and the } \\
\text { ELP3 deletion }\end{array}$ & $\begin{array}{l}\text { Correlation } \\
\text { coefficient between } \\
\text { the control and the } \\
\text { HOS } 2 \text { deletion }\end{array}$ & $\begin{array}{l}\text { Translational } \\
\text { start site }\end{array}$ & $\begin{array}{c}\text { Transcription } \\
\text { direction }\end{array}$ \\
\hline chr07 & YGL180W & 0.982084299 & 0.994334205 & 160071 & + \\
\hline chr07 & $Y G L 171 W$ & 0.969321982 & 0.974163208 & 182396 & + \\
\hline chr07 & $Y G L 163 C$ & 0.975825825 & 0.968348392 & 196409 & - \\
\hline chr07 & YGL138C & 0.971247145 & 0.983410766 & 249536 & - \\
\hline chr07 & YGL120C & 0.981452639 & 0.964320425 & 283943 & - \\
\hline chr07 & YGL119W & 0.986610225 & 0.981525372 & 284448 & + \\
\hline chr07 & YGL108C & 0.961840326 & 0.985939242 & 304074 & - \\
\hline chr07 & YGL058W & 0.977732494 & 0.975176081 & 393992 & + \\
\hline chr07 & YGL056C & 0.960426378 & 0.969119302 & 397624 & - \\
\hline chr07 & YGL055W & 0.964044825 & 0.969447792 & 398631 & + \\
\hline chr07 & YGL048C & 0.971402915 & 0.953304338 & 411289 & - \\
\hline chr07 & YGL043W & 0.964287661 & 0.976335397 & 417487 & + \\
\hline chr07 & YGL028C & 0.960077919 & 0.965512687 & 442914 & - \\
\hline chr07 & YGL006W & 0.950151816 & 0.968357705 & 485925 & + \\
\hline chr07 & YGR001C & 0.958621356 & 0.990717019 & 498038 & - \\
\hline chr07 & YGR006W & 0.980877592 & 0.97721459 & 506074 & + \\
\hline chr07 & YGR027W-B & 0.973585999 & 0.969942324 & 536061 & + \\
\hline chr07 & YGR027W-A & 0.973585999 & 0.969942324 & 536061 & + \\
\hline chr07 & YGR054W & 0.964434273 & 0.980689617 & 596697 & + \\
\hline chr07 & YGR076C & 0.981588154 & 0.958402031 & 637581 & - \\
\hline chr07 & YGR082W & 0.986433799 & 0.977608093 & 644048 & + \\
\hline chr07 & YGR084C & 0.952879595 & 0.959716787 & 648146 & - \\
\hline chr07 & YGR109C & 0.967037071 & 0.989224447 & 706505 & - \\
\hline chr07 & YGR109W-B & 0.964433838 & 0.990506548 & 707614 & + \\
\hline chr07 & YGR109W-A & 0.964433838 & 0.990506548 & 707614 & + \\
\hline chr07 & YGR149W & 0.957244122 & 0.960107682 & 789036 & + \\
\hline chr07 & YGR161W-B & 0.958455297 & 0.972873884 & 811743 & + \\
\hline chr07 & YGR161W-A & 0.958455297 & 0.9728739 & 811743 & + \\
\hline chr07 & YGR161C-D & 0.9711882 & 0.974318881 & 823020 & - \\
\hline chr07 & YGR161C-C & 0.9711882 & 0.9743189 & 823020 & - \\
\hline chr07 & YGR162W & 0.967152562 & 0.972476532 & 824064 & + \\
\hline $\operatorname{chr} 07$ & YGR165W & 0.959888658 & 0.951709091 & 829121 & + \\
\hline chr07 & YGR166W & 0.978481923 & 0.950610672 & 830520 & + \\
\hline chr07 & YGR173W & 0.978946749 & 0.974220242 & 843859 & + \\
\hline chr07 & $Y G R 178 C$ & 0.97338186 & 0.951056442 & 853220 & - \\
\hline chr07 & YGR193C & 0.977703185 & 0.9816188 & 885746 & - \\
\hline chr07 & YGR198W & 0.954889766 & 0.972754857 & 894698 & + \\
\hline chr07 & YGR239C & 0.968888325 & 0.989258725 & 970058 & - \\
\hline chr07 & YGR240C & 0.966313371 & 0.971843468 & 973739 & - \\
\hline chr07 & YGR255C & 0.966583058 & 0.991666026 & 1003967 & - \\
\hline chr07 & YGR267C & 0.960278778 & 0.969707488 & 1025741 & - \\
\hline chr07 & YGR280C & 0.961084505 & 0.952670993 & 1051732 & - \\
\hline chr07 & YGR295C & 0.967286944 & 0.964457386 & 1082736 & - \\
\hline chr07 & YGR296W & 0.971130914 & 0.991185295 & 1084871 & + \\
\hline chr08 & YHLO44W & 0.958725508 & 0.965538017 & 13563 & + \\
\hline chr08 & YHL029C & 0.988947037 & 0.966760586 & 47966 & - \\
\hline chr08 & YHL028W & 0.983034685 & 0.977043411 & 48761 & + \\
\hline chr08 & YHLO24W & 0.961645582 & 0.966633627 & 56647 & + \\
\hline
\end{tabular}


TABle 3: Continued.

\begin{tabular}{|c|c|c|c|c|c|}
\hline Chromosome & Gene & $\begin{array}{l}\text { Correlation } \\
\text { coefficient between } \\
\text { the control and the } \\
\text { ELP3 deletion }\end{array}$ & $\begin{array}{l}\text { Correlation } \\
\text { coefficient between } \\
\text { the control and the } \\
\text { HOS } 2 \text { deletion }\end{array}$ & $\begin{array}{l}\text { Translational } \\
\text { start site }\end{array}$ & $\begin{array}{c}\text { Transcription } \\
\text { direction }\end{array}$ \\
\hline chr08 & YHL020C & 0.978013871 & 0.986901259 & 67453 & - \\
\hline chr08 & YHL016C & 0.976082816 & 0.988408432 & 74241 & - \\
\hline chr08 & YHL007C & 0.973847748 & 0.981293306 & 97933 & - \\
\hline chr08 & YHL004W & 0.97418766 & 0.993677693 & 99215 & + \\
\hline chr08 & YHL001W & 0.959021584 & 0.965334128 & 104272 & + \\
\hline chr08 & YHR001W-A & 0.991644256 & 0.988918444 & 107821 & + \\
\hline chr08 & YHR056C & 0.972836967 & 0.982728846 & 217836 & - \\
\hline chr08 & YHR081W & 0.961720079 & 0.960688905 & 267540 & + \\
\hline chr08 & YHR091C & 0.955695121 & 0.987437891 & 286772 & - \\
\hline chr08 & YHR101C & 0.984820398 & 0.996893941 & 315971 & - \\
\hline chr08 & YHR102W & 0.96127201 & 0.995534701 & 316575 & + \\
\hline chr08 & YHR107C & 0.950250496 & 0.982165489 & 328039 & - \\
\hline chr08 & YHR118C & 0.973725305 & 0.961118169 & 345631 & - \\
\hline chr08 & YHR127W & 0.95014104 & 0.981438554 & 360916 & + \\
\hline chr08 & YHR136C & 0.953476026 & 0.965665058 & 375103 & - \\
\hline chr08 & YHR148W & 0.978585198 & 0.950179497 & 393537 & + \\
\hline chr08 & YHR153C & 0.959414518 & 0.982780385 & 402685 & - \\
\hline chr08 & YHR165C & 0.958747068 & 0.984253541 & 436950 & - \\
\hline chr08 & YHR214C-D & 0.987951207 & 0.966688891 & 550941 & - \\
\hline chr08 & YHR215W & 0.987951207 & 0.966688891 & 552099 & + \\
\hline chr08 & YHR216W & 0.972948554 & 0.975124764 & 554396 & + \\
\hline chr09 & YIL158W & 0.961579117 & 0.957386446 & 46201 & + \\
\hline chr09 & YIL154C & 0.954401073 & 0.971892535 & 55021 & - \\
\hline chr09 & YIL137C & 0.976010283 & 0.976810775 & 92788 & - \\
\hline chr09 & YIL135C & 0.976089791 & 0.979613921 & 96375 & - \\
\hline chr09 & YIL134W & 0.974541094 & 0.979148771 & 97395 & + \\
\hline chr09 & YIL129C & 0.958227819 & 0.99048832 & 113237 & - \\
\hline chr09 & YIL125W & 0.963070211 & 0.960333013 & 122689 & + \\
\hline chr09 & YIL063C & 0.970956253 & 0.957414461 & 243741 & - \\
\hline chr09 & YIL061C & 0.95394965 & 0.989156182 & 245556 & - \\
\hline chr09 & YIL046W & 0.967209958 & 0.952996831 & 268650 & + \\
\hline chr09 & YIL033C & 0.989017477 & 0.989306501 & 291668 & - \\
\hline chr09 & YIL031W & 0.985799922 & 0.98683422 & 292632 & + \\
\hline chr09 & YIL030C & 0.957449018 & 0.97109633 & 300008 & - \\
\hline chr09 & YIR022W & 0.960636707 & 0.971998311 & 398730 & + \\
\hline chr09 & YIR024C & 0.961569219 & 0.970038905 & 403488 & - \\
\hline chr09 & YIR038C & 0.973108358 & 0.975735046 & 424510 & - \\
\hline $\operatorname{chr} 10$ & YJL221C & 0.992889333 & 0.995409763 & 18536 & - \\
\hline $\operatorname{chr} 10$ & YJL219W & 0.992889333 & 0.995409763 & 19497 & + \\
\hline $\operatorname{chr} 10$ & YJL197W & 0.963017116 & 0.967339021 & 63804 & + \\
\hline $\operatorname{chr} 10$ & YJL181W & 0.971567753 & 0.971225117 & 85658 & + \\
\hline $\operatorname{chr} 10$ & YJL176C & 0.979890865 & 0.967535459 & 94528 & - \\
\hline $\operatorname{chr} 10$ & YJL174W & 0.983474082 & 0.9822043 & 95090 & + \\
\hline $\operatorname{chr} 10$ & YJL173C & 0.986867668 & 0.989398406 & 96527 & - \\
\hline $\operatorname{chr} 10$ & YJL151C & 0.952976229 & 0.987631587 & 136770 & - \\
\hline $\operatorname{chr} 10$ & YJL149W & 0.975756648 & 0.990848833 & 137376 & + \\
\hline $\operatorname{chr} 10$ & YJL118W & 0.953268112 & 0.959468203 & 191638 & + \\
\hline $\operatorname{chr} 10$ & YJL113W & 0.982940363 & 0.963188993 & 197913 & + \\
\hline
\end{tabular}


Table 3: Continued.

\begin{tabular}{|c|c|c|c|c|c|}
\hline Chromosome & Gene & $\begin{array}{l}\text { Correlation } \\
\text { coefficient between } \\
\text { the control and the } \\
\text { ELP3 deletion }\end{array}$ & $\begin{array}{l}\text { Correlation } \\
\text { coefficient between } \\
\text { the control and the } \\
\text { HOS } 2 \text { deletion }\end{array}$ & $\begin{array}{l}\text { Translational } \\
\text { start site }\end{array}$ & $\begin{array}{c}\text { Transcription } \\
\text { direction }\end{array}$ \\
\hline chr10 & YJL114W & 0.982940363 & 0.963188993 & 197913 & + \\
\hline chr10 & YJL093C & 0.967675882 & 0.964352732 & 256807 & - \\
\hline chr10 & YJL092W & 0.963240719 & 0.958166876 & 257418 & + \\
\hline chr10 & YJL066C & 0.953982678 & 0.974252054 & 314867 & - \\
\hline chr10 & YJL050W & 0.950677364 & 0.95235067 & 342517 & + \\
\hline chr10 & YJL048C & 0.96132641 & 0.958967756 & 348632 & - \\
\hline chr10 & YJL039C & 0.950918953 & 0.970557445 & 373794 & - \\
\hline $\operatorname{chr} 10$ & YJL034W & 0.963347804 & 0.980266739 & 381322 & + \\
\hline chr10 & YJR010W & 0.971667854 & 0.97428271 & 456232 & + \\
\hline $\operatorname{chr} 10$ & YJR021C & 0.956762721 & 0.994264722 & 469572 & - \\
\hline $\operatorname{chr} 10$ & YJR029W & 0.958070547 & 0.974229894 & 478337 & + \\
\hline chr10 & YJR028W & 0.958070547 & 0.9742299 & 478337 & + \\
\hline chr10 & YJR041C & 0.976618081 & 0.981063923 & 513756 & - \\
\hline chr10 & YJR048W & 0.960606245 & 0.975055837 & 526328 & + \\
\hline chr10 & YJR049C & 0.954983464 & 0.986149168 & 528469 & - \\
\hline $\operatorname{chr} 10$ & YJR055W & 0.976932113 & 0.992763445 & 538765 & + \\
\hline $\operatorname{chr} 10$ & YJR065C & 0.975569729 & 0.987649101 & 559151 & - \\
\hline $\operatorname{chr} 10$ & YJR095W & 0.97047495 & 0.971814385 & 609769 & + \\
\hline $\operatorname{chr} 10$ & YJR113C & 0.969228269 & 0.954716018 & 638969 & - \\
\hline $\operatorname{chr} 10$ & YJR115W & 0.952485858 & 0.960980837 & 639936 & + \\
\hline $\operatorname{chr} 10$ & YJR141W & 0.957349189 & 0.956946497 & 695900 & + \\
\hline $\operatorname{chr} 10$ & YJR160C & 0.978268521 & 0.997490962 & 739810 & - \\
\hline $\operatorname{chr} 10$ & YJR161C & 0.965251269 & 0.976575544 & 743993 & - \\
\hline chr11 & YKL191W & 0.950933251 & 0.978993764 & 81040 & + \\
\hline $\operatorname{chr} 11$ & YKL179C & 0.975707433 & 0.967918905 & 112508 & - \\
\hline $\operatorname{chr} 11$ & $Y K L 167 C$ & 0.956085616 & 0.955561088 & 134139 & - \\
\hline $\operatorname{chr} 11$ & $Y K L 165 C$ & 0.960609423 & 0.966621061 & 140696 & - \\
\hline $\operatorname{chr} 11$ & YKL157W & 0.95635277 & 0.9813363 & 154996 & + \\
\hline $\operatorname{chr} 11$ & YKL127W & 0.955985661 & 0.982208081 & 203185 & + \\
\hline chr11 & YKL125W & 0.976449752 & 0.960074911 & 207891 & + \\
\hline $\operatorname{chr} 11$ & $Y K L 113 C$ & 0.971544612 & 0.954413703 & 225519 & - \\
\hline $\operatorname{chr} 11$ & YKL065C & 0.96005541 & 0.969818928 & 316701 & - \\
\hline $\operatorname{chr} 11$ & YKL064W & 0.974644257 & 0.972632809 & 317408 & + \\
\hline $\operatorname{chr} 11$ & YKL059C & 0.957153957 & 0.961241915 & 329087 & - \\
\hline $\operatorname{chr} 11$ & YKL020C & 0.955860253 & 0.977812511 & 401723 & - \\
\hline chr11 & YKL013C & 0.972958978 & 0.953444609 & 417666 & - \\
\hline $\operatorname{chr} 11$ & YKR007W & 0.981387104 & 0.972277081 & 451077 & + \\
\hline $\operatorname{chr} 11$ & YKR024C & 0.971944038 & 0.981215773 & 487015 & - \\
\hline $\operatorname{chr} 11$ & YKR031C & 0.952123552 & 0.969614994 & 506037 & - \\
\hline $\operatorname{chr} 11$ & YKR036C & 0.959340192 & 0.988104612 & 510275 & - \\
\hline $\operatorname{chr} 11$ & YKR041W & 0.982786597 & 0.957524998 & 517840 & + \\
\hline $\operatorname{chr} 11$ & YKR052C & 0.954392373 & 0.966888713 & 533106 & - \\
\hline $\operatorname{chr} 11$ & YKR082W & 0.962931616 & 0.991018501 & 592467 & + \\
\hline $\operatorname{chr} 11$ & YKR084C & 0.953228662 & 0.976903834 & 598532 & - \\
\hline $\operatorname{chr} 11$ & YKR086W & 0.952006608 & 0.976533657 & 599499 & + \\
\hline $\operatorname{chr} 12$ & YLL050C & 0.976179673 & 0.990695339 & 40413 & - \\
\hline $\operatorname{chr} 12$ & YLL002W & 0.972688874 & 0.981122544 & 146290 & + \\
\hline $\operatorname{chr} 12$ & YLR001C & 0.966206724 & 0.98012374 & 153976 & - \\
\hline
\end{tabular}


TABle 3: Continued.

\begin{tabular}{|c|c|c|c|c|c|}
\hline Chromosome & Gene & $\begin{array}{l}\text { Correlation } \\
\text { coefficient between } \\
\text { the control and the } \\
\text { ELP3 deletion }\end{array}$ & $\begin{array}{l}\text { Correlation } \\
\text { coefficient between } \\
\text { the control and the } \\
\text { HOS } 2 \text { deletion }\end{array}$ & $\begin{array}{l}\text { Translational } \\
\text { start site }\end{array}$ & $\begin{array}{c}\text { Transcription } \\
\text { direction }\end{array}$ \\
\hline chr12 & YLR012C & 0.956516633 & 0.962463803 & 170280 & - \\
\hline chr12 & YLR013W & 0.978873849 & 0.985557204 & 171338 & + \\
\hline $\operatorname{chr} 12$ & YLR024C & 0.953442868 & 0.956160837 & 193282 & - \\
\hline $\operatorname{chr} 12$ & YLR025W & 0.971468039 & 0.96202948 & 194453 & + \\
\hline chr12 & YLR029C & 0.961637928 & 0.991619508 & 202591 & - \\
\hline $\operatorname{chr} 12$ & YLR059C & 0.963450626 & 0.974770886 & 260548 & - \\
\hline chr12 & YLR085C & 0.9560163 & 0.984640913 & 301990 & - \\
\hline $\operatorname{chr} 12$ & YLR087C & 0.953704478 & 0.990085627 & 315732 & - \\
\hline $\operatorname{chr} 12$ & YLR096W & 0.977966477 & 0.979318401 & 332591 & + \\
\hline $\operatorname{chr} 12$ & YLR104W & 0.966174553 & 0.97921157 & 346586 & + \\
\hline $\operatorname{chr} 12$ & YLR133W & 0.961307333 & 0.951823465 & 408446 & + \\
\hline chr12 & YLR135W & 0.977560494 & 0.977991425 & 413282 & + \\
\hline chr12 & YLR137W & 0.967174193 & 0.983596278 & 417007 & + \\
\hline chr12 & YLR162W-A & 0.968123219 & 0.962766706 & 490407 & + \\
\hline $\operatorname{chr} 12$ & YLR208W & 0.96802684 & 0.969296393 & 559553 & + \\
\hline $\operatorname{chr} 12$ & YLR223C & 0.966783974 & 0.974989936 & 585492 & - \\
\hline $\operatorname{chr} 12$ & YLR224W & 0.974582795 & 0.978003757 & 586466 & + \\
\hline $\operatorname{chr} 12$ & YLR286C & 0.972231318 & 0.978358686 & 710138 & - \\
\hline $\operatorname{chr} 12$ & YLR299W & 0.962625418 & 0.970226227 & 726071 & + \\
\hline $\operatorname{chr} 12$ & YLR307W & 0.962710223 & 0.990591743 & 745622 & + \\
\hline $\operatorname{chr} 12$ & YLR323C & 0.953205594 & 0.975247431 & 778952 & - \\
\hline $\operatorname{chr} 12$ & YLR326W & 0.979769576 & 0.991583861 & 782174 & + \\
\hline $\operatorname{chr} 12$ & YLR355C & 0.967858474 & 0.973834311 & 839252 & - \\
\hline $\operatorname{chr} 12$ & YLR356W & 0.968277882 & 0.974767083 & 840320 & + \\
\hline $\operatorname{chr} 12$ & YLR378C & 0.974122358 & 0.977246394 & 877177 & - \\
\hline $\operatorname{chr} 12$ & YLR380W & 0.973258508 & 0.97821865 & 878282 & + \\
\hline $\operatorname{chr} 12$ & YLR410W-A & 0.951545011 & 0.9529714 & 941481 & + \\
\hline $\operatorname{chr} 12$ & $Y L R 410 W-B$ & 0.951545011 & 0.952971407 & 941481 & + \\
\hline $\operatorname{chr} 12$ & YLR426W & 0.9668981 & 0.990317101 & 987059 & + \\
\hline $\operatorname{chr} 12$ & YLR427W & 0.968631588 & 0.972538491 & 988425 & + \\
\hline $\operatorname{chr} 12$ & YLR429W & 0.950464544 & 0.951528134 & 990774 & + \\
\hline $\operatorname{chr} 12$ & YLR443W & 0.964525656 & 0.991440098 & 1022622 & + \\
\hline $\operatorname{chr} 13$ & $Y M L 121 W$ & 0.974659011 & 0.985969584 & 26930 & + \\
\hline $\operatorname{chr} 13$ & $Y M L 115 C$ & 0.97337994 & 0.996063492 & 41794 & - \\
\hline $\operatorname{chr} 13$ & YML080W & 0.989302036 & 0.986381735 & 108806 & + \\
\hline $\operatorname{chr} 13$ & YML078W & 0.975873108 & 0.974136336 & 111002 & + \\
\hline chr13 & $Y M L 045 W$ & 0.993016416 & 0.993250991 & 184461 & + \\
\hline $\operatorname{chr} 13$ & $Y M L 045 W-A$ & 0.993016416 & 0.993251 & 184461 & + \\
\hline $\operatorname{chr} 13$ & YML041C & 0.969801821 & 0.979224272 & 195755 & - \\
\hline $\operatorname{chr} 13$ & YML020W & 0.975260683 & 0.95439232 & 231149 & + \\
\hline $\operatorname{chr} 13$ & YML004C & 0.966616377 & 0.98388072 & 262685 & - \\
\hline $\operatorname{chr} 13$ & $Y M L 003 W$ & 0.968338912 & 0.981828638 & 263483 & + \\
\hline $\operatorname{chr} 13$ & YMR010W & 0.985774326 & 0.979191015 & 285099 & + \\
\hline $\operatorname{chr} 13$ & YMR011W & 0.963732981 & 0.981283867 & 288078 & + \\
\hline $\operatorname{chr} 13$ & YMR027W & 0.961041993 & 0.957831173 & 325876 & + \\
\hline $\operatorname{chr} 13$ & YMR036C & 0.969260192 & 0.963784402 & 343519 & - \\
\hline $\operatorname{chr} 13$ & YMR060C & 0.990018805 & 0.990517987 & 392514 & - \\
\hline $\operatorname{chr} 13$ & YMR066W & 0.98321165 & 0.95995151 & 401540 & + \\
\hline
\end{tabular}


Table 3: Continued.

\begin{tabular}{|c|c|c|c|c|c|}
\hline Chromosome & Gene & $\begin{array}{l}\text { Correlation } \\
\text { coefficient between } \\
\text { the control and the } \\
\text { ELP3 deletion }\end{array}$ & $\begin{array}{l}\text { Correlation } \\
\text { coefficient between } \\
\text { the control and the } \\
\text { HOS } 2 \text { deletion }\end{array}$ & $\begin{array}{l}\text { Translational } \\
\text { start site }\end{array}$ & $\begin{array}{c}\text { Transcription } \\
\text { direction }\end{array}$ \\
\hline chr13 & YMR078C & 0.969392941 & 0.976546196 & 424727 & - \\
\hline chr13 & YMR081C & 0.981254312 & 0.956294421 & 431094 & - \\
\hline chr13 & $Y M R 110 C$ & 0.961424621 & 0.983151943 & 491991 & - \\
\hline chr13 & YMR116C & 0.977129849 & 0.989254306 & 500687 & - \\
\hline chr13 & YMR137C & 0.971971845 & 0.978526214 & 544962 & - \\
\hline chr13 & YMR138W & 0.978634859 & 0.963362392 & 545154 & + \\
\hline chr13 & YMR152W & 0.958913422 & 0.961601086 & 563095 & + \\
\hline $\operatorname{chr} 13$ & YMR197C & 0.960464224 & 0.981756931 & 659197 & - \\
\hline $\operatorname{chr} 13$ & YMR210W & 0.974579381 & 0.983178959 & 687515 & + \\
\hline $\operatorname{chr} 13$ & $Y M R 214 W$ & 0.977906077 & 0.988639434 & 695349 & + \\
\hline $\operatorname{chr} 13$ & $Y M R 219 W$ & 0.964555974 & 0.962486321 & 707132 & + \\
\hline chr13 & $Y M R 224 C$ & 0.975063127 & 0.950620617 & 720652 & - \\
\hline chr13 & YMR229C & 0.960514554 & 0.957025636 & 731122 & - \\
\hline chr13 & YMR241W & 0.956225901 & 0.967912184 & 751960 & + \\
\hline chr13 & YMR319C & 0.950340769 & 0.968043162 & 914536 & - \\
\hline $\operatorname{chr} 14$ & YNL339C & 0.975221324 & 0.997373072 & 6098 & - \\
\hline chr14 & $Y N L 334 C$ & 0.968865734 & 0.991228175 & 12876 & - \\
\hline $\operatorname{chr} 14$ & YNL322C & 0.954706246 & 0.977799203 & 34234 & - \\
\hline $\operatorname{chr} 14$ & YNL311C & 0.986814003 & 0.993782307 & 51687 & - \\
\hline $\operatorname{chr} 14$ & YNL310C & 0.956852702 & 0.980516471 & 52430 & - \\
\hline $\operatorname{chr} 14$ & YNL309W & 0.988062215 & 0.993825499 & 52661 & + \\
\hline $\operatorname{chr} 14$ & YNL301C & 0.968107997 & 0.966680876 & 64562 & - \\
\hline $\operatorname{chr} 14$ & YNL295W & 0.952849161 & 0.964836983 & 76946 & + \\
\hline chr14 & $Y N L 261 W$ & 0.950477134 & 0.976226949 & 155101 & + \\
\hline $\operatorname{chr} 14$ & YNL260C & 0.968893453 & 0.965562883 & 157456 & - \\
\hline $\operatorname{chr} 14$ & YNL255C & 0.959418743 & 0.961868386 & 167791 & - \\
\hline chr14 & $Y N L 248 C$ & 0.959305496 & 0.962132738 & 182609 & - \\
\hline $\operatorname{chr} 14$ & YNL241C & 0.952591706 & 0.968306617 & 197944 & - \\
\hline $\operatorname{chr} 14$ & YNL234W & 0.975005906 & 0.964281927 & 210234 & + \\
\hline $\operatorname{chr} 14$ & YNL224C & 0.973438019 & 0.985601741 & 227100 & - \\
\hline $\operatorname{chr} 14$ & $Y N L 212 W$ & 0.967303893 & 0.964441355 & 247462 & + \\
\hline $\operatorname{chr} 14$ & $Y N L 166 C$ & 0.973079673 & 0.979903307 & 323567 & - \\
\hline $\operatorname{chr} 14$ & $Y N L 156 C$ & 0.961076874 & 0.96913283 & 341970 & - \\
\hline $\operatorname{chr} 14$ & $Y N L 112 W$ & 0.953769466 & 0.987266001 & 413641 & + \\
\hline $\operatorname{chr} 14$ & YNL099C & 0.959678116 & 0.95727388 & 439285 & - \\
\hline $\operatorname{chr} 14$ & YNL097C & 0.978605808 & 0.986440434 & 442360 & - \\
\hline $\operatorname{chr} 14$ & YNL082W & 0.955790378 & 0.980457797 & 473392 & + \\
\hline chr14 & YNL055C & 0.982685169 & 0.981068004 & 518846 & - \\
\hline $\operatorname{chr} 14$ & $Y N L 042 W-B$ & 0.956836355 & 0.979900708 & 547114 & + \\
\hline $\operatorname{chr} 14$ & YNL029C & 0.976101029 & 0.988675718 & 578774 & - \\
\hline $\operatorname{chr} 14$ & YNL027W & 0.96462873 & 0.978413199 & 579581 & + \\
\hline chr14 & YNR012W & 0.958400399 & 0.977490746 & 647434 & + \\
\hline chr14 & YNR015W & 0.967843094 & 0.979265652 & 653389 & + \\
\hline $\operatorname{chr} 14$ & YNR023W & 0.975180387 & 0.972628935 & 670420 & + \\
\hline $\operatorname{chr} 14$ & YNR026C & 0.966267575 & 0.984095961 & 674691 & - \\
\hline chr14 & YNR036C & 0.955455936 & 0.956914254 & 694824 & - \\
\hline $\operatorname{chr} 14$ & YNR039C & 0.97056523 & 0.9708253 & 699433 & - \\
\hline chr14 & YNR075W & 0.950188074 & 0.953766447 & 779916 & + \\
\hline
\end{tabular}


TABle 3: Continued.

\begin{tabular}{|c|c|c|c|c|c|}
\hline Chromosome & Gene & $\begin{array}{l}\text { Correlation } \\
\text { coefficient between } \\
\text { the control and the } \\
\text { ELP3 deletion }\end{array}$ & $\begin{array}{l}\text { Correlation } \\
\text { coefficient between } \\
\text { the control and the } \\
\text { HOS } 2 \text { deletion }\end{array}$ & $\begin{array}{l}\text { Translational } \\
\text { start site }\end{array}$ & $\begin{array}{c}\text { Transcription } \\
\text { direction }\end{array}$ \\
\hline chr14 & YNR075C-A & 0.986389631 & 0.991000914 & 781603 & - \\
\hline chr15 & $Y O L 166 W-A$ & 0.969218719 & 0.964760148 & 585 & + \\
\hline chr15 & YOL157C & 0.977029388 & 0.98945557 & 24293 & - \\
\hline chr15 & YOL156W & 0.979765672 & 0.992685071 & 25272 & + \\
\hline chr15 & $Y O L 148 C$ & 0.957761501 & 0.971157209 & 47573 & - \\
\hline chr15 & $Y O L 104 C$ & 0.976917372 & 0.981386975 & 117454 & - \\
\hline chr15 & YOL100W & 0.961328138 & 0.959394148 & 129237 & + \\
\hline $\operatorname{chr} 15$ & YOL089C & 0.971692903 & 0.984860678 & 153490 & - \\
\hline chr15 & YOL086C & 0.976082094 & 0.966862518 & 160594 & - \\
\hline $\operatorname{chr} 15$ & YOL077C & 0.956963602 & 0.983941333 & 186723 & - \\
\hline $\operatorname{chr} 15$ & YOL068C & 0.959180595 & 0.953847182 & 201879 & - \\
\hline chr15 & YOL062C & 0.954102242 & 0.967526899 & 211995 & - \\
\hline chr15 & YOL058W & 0.969401099 & 0.978364009 & 219210 & + \\
\hline chr15 & YOL045W & 0.966108727 & 0.955785397 & 243496 & + \\
\hline chr15 & YOL031C & 0.971563515 & 0.959211572 & 267530 & - \\
\hline $\operatorname{chr} 15$ & YOL026C & 0.954115513 & 0.976281121 & 274354 & - \\
\hline chr15 & YOL023W & 0.960879668 & 0.960550998 & 278057 & + \\
\hline $\operatorname{chr} 15$ & YOL006C & 0.971429281 & 0.978082289 & 315388 & - \\
\hline chr15 & YOR043W & 0.970409112 & 0.977199399 & 410870 & + \\
\hline chr15 & YOR048C & 0.95240535 & 0.972598244 & 421651 & - \\
\hline chr15 & YOR056C & 0.95552512 & 0.980531823 & 431628 & - \\
\hline $\operatorname{chr} 15$ & YOR058C & 0.977288174 & 0.978577416 & 436347 & - \\
\hline $\operatorname{chr} 15$ & YOR071C & 0.980341941 & 0.989920087 & 461278 & - \\
\hline chr15 & YOR075W & 0.983591979 & 0.981471486 & 468214 & + \\
\hline $\operatorname{chr} 15$ & YOR089C & 0.978934603 & 0.984066065 & 490830 & - \\
\hline $\operatorname{chr} 15$ & YOR104W & 0.965107091 & 0.973591736 & 517643 & + \\
\hline $\operatorname{chr} 15$ & YOR124C & 0.97179062 & 0.983946384 & 558643 & - \\
\hline $\operatorname{chr} 15$ & YOR129C & 0.979558976 & 0.983142651 & 569559 & - \\
\hline $\operatorname{chr} 15$ & YOR132W & 0.955282168 & 0.965555781 & 573176 & + \\
\hline chr15 & YOR148C & 0.987044445 & 0.98569999 & 609198 & - \\
\hline $\operatorname{chr} 15$ & YOR192C-C & 0.980704362 & 0.966062169 & 704225 & - \\
\hline chr15 & YOR193W & 0.973179583 & 0.992657727 & 710447 & + \\
\hline $\operatorname{chr} 15$ & YOR204W & 0.985619071 & 0.960639074 & 722912 & + \\
\hline chr15 & YOR216C & 0.971045967 & 0.970515948 & 748980 & - \\
\hline chr15 & YOR247W & 0.959336754 & 0.957210052 & 797677 & + \\
\hline $\operatorname{chr} 15$ & YOR294W & 0.972999786 & 0.97236413 & 868339 & + \\
\hline chr15 & YOR336W & 0.973501735 & 0.969442343 & 949770 & + \\
\hline $\operatorname{chr} 15$ & YOR365C & 0.981766719 & 0.98630012 & 1025570 & - \\
\hline $\operatorname{chr} 15$ & YOR372C & 0.955936893 & 0.971693401 & 1036469 & - \\
\hline $\operatorname{chr} 15$ & YOR389W & 0.964833697 & 0.976852438 & 1074211 & + \\
\hline $\operatorname{chr} 15$ & YOR390W & 0.98673199 & 0.997343789 & 1076782 & + \\
\hline $\operatorname{chr} 15$ & YOR391C & 0.952090431 & 0.990889663 & 1079256 & - \\
\hline $\operatorname{chr} 15$ & YOR393W & 0.952090431 & 0.990889663 & 1080274 & + \\
\hline chr16 & YPL283C & 0.990485958 & 0.9953985 & 6007 & - \\
\hline chr16 & YPL281C & 0.961921468 & 0.977623026 & 10870 & - \\
\hline chr16 & YPL280W & 0.961921468 & 0.977623026 & 11887 & + \\
\hline $\operatorname{chr} 16$ & YPL278C & 0.965633274 & 0.959753752 & 15355 & - \\
\hline $\operatorname{chr} 16$ & YPL257W-B & 0.976897418 & 0.976806903 & 56748 & + \\
\hline
\end{tabular}


Table 3: Continued.

\begin{tabular}{|c|c|c|c|c|c|}
\hline Chromosome & Gene & $\begin{array}{l}\text { Correlation } \\
\text { coefficient between } \\
\text { the control and the } \\
\text { ELP3 deletion }\end{array}$ & $\begin{array}{l}\text { Correlation } \\
\text { coefficient between } \\
\text { the control and the } \\
\text { HOS } 2 \text { deletion }\end{array}$ & $\begin{array}{l}\text { Translational } \\
\text { start site }\end{array}$ & $\begin{array}{c}\text { Transcription } \\
\text { direction }\end{array}$ \\
\hline chr16 & $Y P L 257 W-A$ & 0.976897418 & 0.976807 & 56748 & + \\
\hline chr16 & YPL255W & 0.96664841 & 0.970266728 & 67725 & + \\
\hline chr16 & YPL254W & 0.95123182 & 0.962107221 & 69485 & + \\
\hline $\operatorname{chr} 16$ & YPL206C & 0.975807996 & 0.972912432 & 163596 & - \\
\hline chr16 & YPL196W & 0.978338347 & 0.984467392 & 175042 & + \\
\hline chr16 & YPL180W & 0.976152618 & 0.987674239 & 205247 & + \\
\hline chr16 & YPL171C & 0.96524809 & 0.961521166 & 227370 & - \\
\hline chr16 & YPL158C & 0.980225654 & 0.985491741 & 254309 & - \\
\hline chr16 & YPL157W & 0.98638216 & 0.988124386 & 254813 & + \\
\hline chr16 & YPL146C & 0.98509812 & 0.956540524 & 277528 & - \\
\hline $\operatorname{chr} 16$ & YPL139C & 0.981115598 & 0.987215069 & 291050 & - \\
\hline chr16 & YPL126W & 0.988937478 & 0.982334739 & 310209 & + \\
\hline chr16 & YPL082C & 0.980458525 & 0.992300343 & 404080 & - \\
\hline $\operatorname{chr} 16$ & YPL078C & 0.957476311 & 0.95576197 & 408741 & - \\
\hline chr16 & YPL038W & 0.953416137 & 0.966495031 & 480532 & + \\
\hline chr16 & YPL030W & 0.973101156 & 0.975002429 & 493541 & + \\
\hline $\operatorname{chr} 16$ & YPL007C & 0.954338314 & 0.983931954 & 543845 & - \\
\hline chr16 & YPR010C & 0.983770387 & 0.983449754 & 581193 & - \\
\hline chr16 & YPR017C & 0.986325514 & 0.989002388 & 593914 & - \\
\hline $\operatorname{chr} 16$ & YPR018W & 0.981145288 & 0.96510122 & 594473 & + \\
\hline chr16 & YPR020W & 0.950323294 & 0.983815562 & 599867 & + \\
\hline chr16 & YPR048W & 0.980910651 & 0.976100682 & 659179 & + \\
\hline chr16 & YPR060C & 0.960902075 & 0.969265445 & 675628 & - \\
\hline chr16 & YPR062W & 0.96911875 & 0.967204709 & 677162 & + \\
\hline chr16 & YPR088C & 0.957617837 & 0.976321629 & 713026 & - \\
\hline chr16 & YPR141C & 0.978516693 & 0.973377917 & 817919 & - \\
\hline chr16 & YPR145C-A & 0.961127516 & 0.965220022 & 824922 & - \\
\hline chr16 & YPR156C & 0.966866802 & 0.956230015 & 839773 & - \\
\hline $\operatorname{chr} 16$ & YPR158C-D & 0.981640164 & 0.971311933 & 856253 & - \\
\hline chr16 & YPR158C-C & 0.981640164 & 0.971311933 & 856253 & - \\
\hline $\operatorname{chr} 16$ & YPR161C & 0.969622155 & 0.984283791 & 866418 & - \\
\hline $\operatorname{chr} 16$ & YPR165W & 0.959239007 & 0.967322323 & 875364 & + \\
\hline chr16 & YPR176C & 0.986542093 & 0.977514778 & 892074 & - \\
\hline $\operatorname{chr} 16$ & YPR187W & 0.964480751 & 0.98804279 & 911253 & + \\
\hline chr16 & YPR203W & 0.968955474 & 0.985644074 & 943876 & + \\
\hline
\end{tabular}

on genome-wide nucleosome mapping data for $A$. fumigatus [9] and S. cerevisiae [10]. In this analysis, a $1-\mathrm{kb}$ region upstream of the translational start site was defined as a gene promoter. When the length of the gene body region is more than $1 \mathrm{~kb}$, a $1-\mathrm{kb}$ region downstream of the translational start site was defined as the gene body. When the length of the gene body is less than $1 \mathrm{~kb}$, the region between the translational start and end sites was defined as the gene body. Analyses of nucleosome position data including calculation of Spearman's rank correlation coefficient were performed using the statistics software R (http://www.r-project.org/).

\section{Results and Discussion}

3.1. Nucleosome Position Profiles of Duplicated Genes in Aspergillus fumigatus. I compared nucleosome position profiles in each of the 63 duplicated gene pairs. Nucleosome positioning was conserved more in gene promoters than in gene bodies (Figure 1), as observed in the comparison of nucleosome positioning between trichostatin A-treated and -untreated A. fumigatus [4]. This result suggests that nucleosome positioning in the gene promoter plays an important role in transcriptional regulation [14]. 
TABLE 4: Spearman's rank correlation coefficients between nucleosome position profiles in the promoters of 347 orthologous gene pairs between Aspergillus fumigatus and Saccharomyces cerevisiae.

\begin{tabular}{|c|c|c|}
\hline S. cerevisiae gene & A. fumigatus gene & Correlation coefficient \\
\hline YCR053W & AFUA_3G08980 & 0.851750303 \\
\hline YOL006C & AFUA_1G03500 & 0.814728478 \\
\hline YLR208W & AFUA_4G06090 & 0.810778356 \\
\hline YJR160C & AFUA_8G07240 & 0.807607644 \\
\hline YKL064W & AFUA_2G08070 & 0.771984405 \\
\hline YDL208W & AFUA_1G13570 & 0.763687527 \\
\hline YDL247W & AFUA_8G07240 & 0.751799318 \\
\hline YJL034W & AFUA_2G04620 & 0.751475734 \\
\hline YJL174W & AFUA_2G07590 & 0.731359519 \\
\hline YGL006W & AFUA_1G10880 & 0.706118907 \\
\hline YLR307W & AFUA_6G10430 & 0.701428537 \\
\hline YOR056C & AFUA_5G04000 & 0.693624039 \\
\hline YJR049C & AFUA_5G12870 & 0.6873278 \\
\hline YOR132W & AFUA_5G07150 & 0.684358232 \\
\hline YDR028C & AFUA_8G02720 & 0.681519128 \\
\hline YKL059C & AFUA_2G06220 & 0.677236775 \\
\hline YDR019C & AFUA_1G10780 & 0.673590566 \\
\hline YDR262W & AFUA_8G05360 & 0.668577442 \\
\hline YJR160C & AFUA_3G01700 & 0.655149877 \\
\hline YBL087C & AFUA_2G03380 & 0.654275917 \\
\hline YDR120C & AFUA_3G04200 & 0.653965099 \\
\hline YMR110C & AFUA_4G13500 & 0.649573592 \\
\hline YDR479C & AFUA_2G01510 & 0.649340576 \\
\hline YLR307W & AFUA_4G09940 & 0.641152119 \\
\hline YLL050C & AFUA_5G10570 & 0.636927762 \\
\hline YMR011W & AFUA_2G11520 & 0.631877767 \\
\hline YGR149W & AFUA_3G08240 & 0.627253467 \\
\hline YKL125W & AFUA_1G02590 & 0.626251676 \\
\hline YDL247W & AFUA_3G01700 & 0.621782331 \\
\hline YHR165C & AFUA_2G03030 & 0.616638486 \\
\hline YLR355C & AFUA_3G14490 & 0.615946987 \\
\hline YLR307W & AFUA_3G07210 & 0.615345397 \\
\hline YNL156C & AFUA_4G07680 & 0.605449075 \\
\hline YOL157C & AFUA_8G07070 & 0.600254438 \\
\hline YPL196W & AFUA_3G08740 & 0.596649007 \\
\hline YOL156W & AFUA_2G11520 & 0.589475104 \\
\hline YGL055W & AFUA_7G05920 & 0.583471232 \\
\hline YER094C & AFUA_4G07420 & 0.580111415 \\
\hline YER026C & AFUA_4G13680 & 0.579632053 \\
\hline YKR031C & AFUA_3G05630 & 0.578947614 \\
\hline YJR041C & AFUA_1G13200 & 0.577042969 \\
\hline YCL057C-A & AFUA_1G13195 & 0.56703028 \\
\hline YKL167C & AFUA_6G12620 & 0.566497151 \\
\hline YDR334W & AFUA_7G02370 & 0.556810391 \\
\hline YDL085W & AFUA_1G11960 & 0.555058025 \\
\hline YIR038C & AFUA_8G02500 & 0.553657653 \\
\hline YGL056C & AFUA_1G06660 & 0.550891198 \\
\hline YLR133W & AFUA_1G15930 & 0.547961885 \\
\hline YLR137W & AFUA_2G09930 & 0.542448721 \\
\hline
\end{tabular}


Table 4: Continued.

\begin{tabular}{|c|c|c|}
\hline S. cerevisiae gene & A. fumigatus gene & Correlation coefficient \\
\hline YER095W & AFUA_1G10410 & 0.540102076 \\
\hline YCR090C & AFUA_2G15510 & 0.540015424 \\
\hline YIR022W & AFUA_3G12840 & 0.539096148 \\
\hline YJR048W & AFUA_2G13110 & 0.537701308 \\
\hline YDL247W & AFUA_8G01340 & 0.536753536 \\
\hline YGL119W & AFUA_6G04380 & 0.528264148 \\
\hline YDR054C & AFUA_5G09200 & 0.520282879 \\
\hline YNR036C & AFUA_5G10750 & 0.51817226 \\
\hline YJL050W & AFUA_4G07160 & 0.517374235 \\
\hline YKR036C & AFUA_5G13140 & 0.515007541 \\
\hline YML041C & AFUA_2G05030 & 0.514819597 \\
\hline YML080W & AFUA_1G16550 & 0.512494533 \\
\hline YJL093C & AFUA_1G14250 & 0.5107227 \\
\hline$Y D R 238 C$ & AFUA_1G10970 & 0.509749906 \\
\hline YJL197W & AFUA_2G14130 & 0.501902821 \\
\hline YER173W & AFUA_8G02820 & 0.501581347 \\
\hline YJR160C & AFUA_8G01340 & 0.499250408 \\
\hline YOL058W & AFUA_2G04310 & 0.497155736 \\
\hline YGR006W & AFUA_1G16990 & 0.496383573 \\
\hline YPR161C & AFUA_5G05510 & 0.495551014 \\
\hline YBR136W & AFUA_4G04760 & 0.49434371 \\
\hline YJR049C & AFUA_2G01350 & 0.493221085 \\
\hline YPR060C & AFUA_5G13130 & 0.490832735 \\
\hline YDR055W & AFUA_4G06820 & 0.482973721 \\
\hline YLR104W & AFUA_5G04040 & 0.47610876 \\
\hline YIL129C & AFUA_6G11010 & 0.473123981 \\
\hline YDR300C & AFUA_2G07570 & 0.469287567 \\
\hline YOL156W & AFUA_7G00950 & 0.46726022 \\
\hline YGL201C & AFUA_5G10890 & 0.462753539 \\
\hline YKR086W & AFUA_1G03820 & 0.461061937 \\
\hline YPL157W & AFUA_6G08610 & 0.460257721 \\
\hline YIL046W & AFUA_2G14110 & 0.45798838 \\
\hline YGL048C & AFUA_4G04660 & 0.457664607 \\
\hline YHR216W & AFUA_2G03610 & 0.456902272 \\
\hline YDR424C & AFUA_1G04850 & 0.455530713 \\
\hline YPR141C & AFUA_2G14280 & 0.452133975 \\
\hline YLR427W & AFUA_1G07150 & 0.444534443 \\
\hline YHL004W & AFUA_1G06570 & 0.439699159 \\
\hline YLL002W & AFUA_5G09540 & 0.431520497 \\
\hline YKR052C & AFUA_6G12550 & 0.429429023 \\
\hline YNL260C & AFUA_1G09000 & 0.425937756 \\
\hline YNL112W & AFUA_2G10750 & 0.423331447 \\
\hline YFL026W & AFUA_3G14330 & 0.422540136 \\
\hline YCLO38C & AFUA_2G15370 & 0.420548714 \\
\hline YPR156C & AFUA_4G01140 & 0.419838437 \\
\hline YHLO24W & AFUA_3G06230 & 0.417835282 \\
\hline YBR173C & AFUA_5G10740 & 0.414366599 \\
\hline YGR178C & AFUA_1G09630 & 0.410618083 \\
\hline YMR036C & AFUA_6G08200 & 0.408252022 \\
\hline
\end{tabular}


TABle 4: Continued.

\begin{tabular}{|c|c|c|}
\hline S. cerevisiae gene & A. fumigatus gene & Correlation coefficient \\
\hline$Y D L 147 W$ & AFUA_3G06610 & 0.40670988 \\
\hline YJR095W & AFUA_2G16930 & 0.405617695 \\
\hline YPL171C & AFUA_2G17960 & 0.40043227 \\
\hline YBL061C & AFUA_8G05620 & 0.398124519 \\
\hline YHL001W & AFUA_6G03830 & 0.397471165 \\
\hline YNL255C & AFUA_1G07630 & 0.390527675 \\
\hline YGL006W & AFUA_3G10690 & 0.389206341 \\
\hline YIL125W & AFUA_4G11650 & 0.387564721 \\
\hline YMR011W & AFUA_5G01160 & 0.381368332 \\
\hline YMR010W & AFUA_5G03510 & 0.378223656 \\
\hline YMR319C & AFUA_4G14640 & 0.376638524 \\
\hline$Y D L 110 C$ & AFUA_1G09160 & 0.376540694 \\
\hline YOL086C & AFUA_5G06240 & 0.376281416 \\
\hline YGR076C & AFUA_4G09000 & 0.373900773 \\
\hline YOR204W & AFUA_4G07660 & 0.368301524 \\
\hline YMR229C & AFUA_2G16040 & 0.367246087 \\
\hline YLR025W & AFUA_1G06420 & 0.363738278 \\
\hline YPL281C & AFUA_6G06770 & 0.363265962 \\
\hline YOR294W & AFUA_7G04430 & 0.363077313 \\
\hline YLR299W & AFUA_7G04760 & 0.36169498 \\
\hline YCL038C & AFUA_2G06170 & 0.359699115 \\
\hline YKL113C & AFUA_3G06060 & 0.351986208 \\
\hline YHL020C & AFUA_5G09420 & 0.350312962 \\
\hline YMR224C & AFUA_6G11410 & 0.348946198 \\
\hline YDR444W & AFUA_3G04240 & 0.345942811 \\
\hline YER107C & AFUA_1G09020 & 0.34238252 \\
\hline YDR162C & AFUA_2G03680 & 0.342381673 \\
\hline$Y N L 212 W$ & AFUA_3G08750 & 0.341561675 \\
\hline YNL097C & AFUA_3G11940 & 0.339603963 \\
\hline YKL191W & AFUA_6G07100 & 0.337319743 \\
\hline YPL030W & AFUA_6G02570 & 0.336821966 \\
\hline YNL224C & AFUA_3G05330 & 0.336636223 \\
\hline YKR024C & AFUA_5G11050 & 0.328747023 \\
\hline YPR020W & AFUA_1G16280 & 0.323479927 \\
\hline YPL171C & AFUA_2G04060 & 0.322952534 \\
\hline YOR393W & AFUA_6G06770 & 0.321210691 \\
\hline YPR088C & AFUA_5G03880 & 0.31284654 \\
\hline YGL058W & AFUA_6G14210 & 0.312000165 \\
\hline YFL059W & AFUA_5G08090 & 0.309971957 \\
\hline YBR243C & AFUA_2G11240 & 0.307271969 \\
\hline YJL048C & AFUA_3G06360 & 0.306215007 \\
\hline YNL055C & AFUA_4G06910 & 0.305279025 \\
\hline YOR058C & AFUA_2G16260 & 0.305221634 \\
\hline YGR240C & AFUA_4G00960 & 0.304340045 \\
\hline YBR279W & AFUA_2G11000 & 0.303224 \\
\hline YPL078C & AFUA_8G05440 & 0.300712891 \\
\hline YLR059C & AFUA_3G11820 & 0.296403753 \\
\hline YLR380W & AFUA_6G12690 & 0.295562758 \\
\hline$Y D L 174 C$ & AFUA_1G17520 & 0.29517154 \\
\hline
\end{tabular}


Table 4: Continued.

\begin{tabular}{|c|c|c|}
\hline S. cerevisiae gene & A. fumigatus gene & Correlation coefficient \\
\hline YCL057W & AFUA_7G05930 & 0.293762978 \\
\hline YDR234W & AFUA_5G08890 & 0.293096856 \\
\hline YDR438W & AFUA_5G12140 & 0.287954196 \\
\hline YGL028C & AFUA_8G05610 & 0.287899385 \\
\hline YML004C & AFUA_6G07940 & 0.285261452 \\
\hline YOL045W & AFUA_2G02850 & 0.284622252 \\
\hline YLR429W & AFUA_2G14270 & 0.283845667 \\
\hline YDR397C & AFUA_3G02340 & 0.283499213 \\
\hline YBR260C & AFUA_3G06280 & 0.282196097 \\
\hline YCR087C-A & AFUA_7G04700 & 0.281322207 \\
\hline YMR011W & AFUA_7G00950 & 0.280854319 \\
\hline$Y O L 148 C$ & AFUA_1G16580 & 0.274850272 \\
\hline YDR379W & AFUA_1G12680 & 0.273041289 \\
\hline YJL151C & AFUA_5G10590 & 0.272979399 \\
\hline YML020W & AFUA_5G12090 & 0.266526854 \\
\hline YGR082W & AFUA_6G11380 & 0.265129767 \\
\hline YJR113C & AFUA_1G04280 & 0.262789584 \\
\hline YIL031W & AFUA_5G03200 & 0.256820972 \\
\hline YDR432W & AFUA_3G10100 & 0.255963453 \\
\hline YDR359C & AFUA_4G07560 & 0.255317292 \\
\hline YDR233C & AFUA_6G13670 & 0.253861938 \\
\hline YGR255C & AFUA_4G12930 & 0.252312468 \\
\hline YFR009W & AFUA_4G06070 & 0.245534257 \\
\hline YPL280W & AFUA_3G08490 & 0.243781447 \\
\hline YOR075W & AFUA_2G09670 & 0.239487119 \\
\hline YJL219W & AFUA_7G00950 & 0.239124986 \\
\hline YCR026C & AFUA_2G14770 & 0.234276623 \\
\hline YOR391C & AFUA_3G08490 & 0.233098887 \\
\hline YGL180W & AFUA_4G09050 & 0.231299038 \\
\hline YLR323C & AFUA_5G07720 & 0.231114462 \\
\hline YGL006W & AFUA_7G01030 & 0.222088168 \\
\hline YBR179C & AFUA_5G13392 & 0.219453048 \\
\hline YKR082W & AFUA_4G05840 & 0.214144582 \\
\hline YLR087C & AFUA_2G13520 & 0.209434557 \\
\hline YCL064C & AFUA_4G07810 & 0.204494803 \\
\hline YOL156W & AFUA_5G01160 & 0.203744073 \\
\hline YBR249C & AFUA_7G04070 & 0.202306902 \\
\hline YJL093C & AFUA_3G07540 & 0.201465401 \\
\hline YOL077C & AFUA_1G02210 & 0.196710807 \\
\hline YLR426W & AFUA_1G06280 & 0.196041865 \\
\hline YMR060C & AFUA_2G03840 & 0.194299595 \\
\hline YOR048C & AFUA_1G13730 & 0.193994391 \\
\hline$Y D L 174 C$ & AFUA_7G02560 & 0.193117882 \\
\hline YJL221C & AFUA_8G07070 & 0.187015236 \\
\hline YGR054W & AFUA_3G05970 & 0.181434929 \\
\hline YPR176C & AFUA_7G04460 & 0.180215936 \\
\hline$Y M L 003 W$ & AFUA_1G09870 & 0.173517378 \\
\hline YKR084C & AFUA_2G04630 & 0.171646807 \\
\hline YDR270W & AFUA_4G12620 & 0.171601357 \\
\hline
\end{tabular}


Table 4: Continued.

\begin{tabular}{|c|c|c|}
\hline S. cerevisiae gene & A. fumigatus gene & Correlation coefficient \\
\hline YOR336W & AFUA_2G02360 & 0.164107564 \\
\hline YFL058W & AFUA_5G02470 & 0.160695353 \\
\hline YNL301C & AFUA_2G07380 & 0.151927636 \\
\hline YJL221C & AFUA_7G06380 & 0.150927464 \\
\hline YGR280C & AFUA_7G03690 & 0.150422383 \\
\hline YOL089C & AFUA_6G01960 & 0.149731857 \\
\hline YGR193C & AFUA_3G08270 & 0.14875632 \\
\hline$Y D R 328 C$ & AFUA_5G06060 & 0.143711765 \\
\hline YPL254W & AFUA_2G06060 & 0.143220591 \\
\hline YPL206C & AFUA_2G00990 & 0.138505935 \\
\hline YEL021W & AFUA_2G08360 & 0.13720587 \\
\hline YOL068C & AFUA_4G12120 & 0.134069861 \\
\hline YJR160C & AFUA_7G06390 & 0.131179873 \\
\hline YNL310C & AFUA_6G08230 & 0.130305791 \\
\hline YIL061C & AFUA_5G13480 & 0.126828721 \\
\hline YGL163C & AFUA_6G12910 & 0.12597657 \\
\hline YGR173W & AFUA_5G06770 & 0.123606414 \\
\hline YJL092W & AFUA_2G03910 & 0.122708164 \\
\hline YJR065C & AFUA_5G11560 & 0.121854722 \\
\hline YBR023C & AFUA_8G05630 & 0.120791924 \\
\hline YBR084W & AFUA_3G08650 & 0.118154138 \\
\hline YMR197C & AFUA_4G10710 & 0.114428905 \\
\hline YMR241W & AFUA_5G04220 & 0.114113659 \\
\hline YNL097C & AFUA_7G01870 & 0.109316946 \\
\hline YGL171W & AFUA_1G16290 & 0.103112508 \\
\hline YOR365C & AFUA_4G13340 & 0.094260129 \\
\hline YHR215W & AFUA_6G11330 & 0.092128581 \\
\hline YKL013C & AFUA_6G02370 & 0.088958318 \\
\hline YOR043W & AFUA_4G06130 & 0.087990985 \\
\hline YPR062W & AFUA_1G05050 & 0.087018417 \\
\hline YDR497C & AFUA_2G07910 & 0.078863767 \\
\hline YDR477W & AFUA_2G01700 & 0.074801159 \\
\hline YLR378C & AFUA_5G08130 & 0.074624328 \\
\hline YBR060C & AFUA_5G08110 & 0.073589072 \\
\hline YGL120C & AFUA_5G11620 & 0.069009268 \\
\hline YIL063C & AFUA_2G10810 & 0.06328373 \\
\hline YOL157C & AFUA_7G06380 & 0.06047767 \\
\hline YGL043W & AFUA_3G07670 & 0.059398891 \\
\hline YMR027W & AFUA_5G06710 & 0.058232831 \\
\hline YHR215W & AFUA_8G01910 & 0.05787337 \\
\hline$Y D L 247 W$ & AFUA_7G05190 & 0.05184022 \\
\hline YPR048W & AFUA_5G07290 & 0.049809159 \\
\hline YBL051C & AFUA_5G01940 & 0.048514144 \\
\hline YHR148W & AFUA_2G08320 & 0.047441878 \\
\hline YJR160C & AFUA_7G05190 & 0.045923723 \\
\hline YJL039C & AFUA_5G12670 & 0.042943521 \\
\hline YNL029C & AFUA_5G12160 & 0.031203315 \\
\hline YGR267C & AFUA_5G03140 & 0.029526621 \\
\hline YNL027W & AFUA_1G06900 & 0.02747405 \\
\hline
\end{tabular}


Table 4: Continued.

\begin{tabular}{|c|c|c|}
\hline S. cerevisiae gene & A. fumigatus gene & Correlation coefficient \\
\hline YDL247W & AFUA_7G06390 & 0.023887472 \\
\hline YHR107C & AFUA_5G03080 & 0.020068991 \\
\hline$Y K L 165 C$ & AFUA_4G03970 & 0.01938034 \\
\hline YNR012W & AFUA_2G05430 & 0.019075112 \\
\hline YLR085C & AFUA_4G04420 & 0.018617278 \\
\hline YPL082C & AFUA_1G05830 & 0.017285628 \\
\hline YCL064C & AFUA_1G06150 & 0.014476469 \\
\hline YNR039C & AFUA_1G12090 & 0.011820922 \\
\hline YOR216C & AFUA_1G08830 & 0.008439713 \\
\hline YOR148C & AFUA_4G07550 & 0.006702846 \\
\hline YDR420W & AFUA_4G00500 & 0.006566287 \\
\hline YPR018W & AFUA_5G03720 & -0.000683184 \\
\hline YDR322W & AFUA_5G12810 & -0.002517201 \\
\hline YNR015W & AFUA_3G08390 & -0.013154917 \\
\hline YBR251W & AFUA_5G11540 & -0.01637247 \\
\hline YHL028W & AFUA_5G09020 & -0.020657813 \\
\hline YPL146C & AFUA_2G05550 & -0.023787247 \\
\hline YKL127W & AFUA_3G11830 & -0.026619404 \\
\hline YJR160C & AFUA_2G10910 & -0.029508087 \\
\hline YIR038C & AFUA_1G17010 & -0.030714781 \\
\hline YDL247W & AFUA_2G10910 & -0.033905332 \\
\hline YBR204C & AFUA_1G03540 & -0.038361835 \\
\hline YJR010W & AFUA_3G06530 & -0.038908707 \\
\hline YMR210W & AFUA_6G04640 & -0.046477426 \\
\hline YPR187W & AFUA_1G05160 & -0.048502572 \\
\hline YDR037W & AFUA_6G07640 & -0.052027721 \\
\hline YIL134W & AFUA_6G05170 & -0.053118026 \\
\hline YLR286C & AFUA_5G03760 & -0.062116003 \\
\hline YKL179C & AFUA_1G14240 & -0.062203351 \\
\hline YDR109C & AFUA_4G04680 & -0.063717983 \\
\hline YOL157C & AFUA_3G07380 & -0.064624439 \\
\hline YIR038C & AFUA_2G17300 & -0.068756542 \\
\hline YDL102W & AFUA_2G16600 & -0.088010728 \\
\hline YOR389W & AFUA_2G01940 & -0.09375584 \\
\hline YBR244W & AFUA_3G12270 & -0.097477745 \\
\hline YJL219W & AFUA_2G11520 & -0.098366126 \\
\hline YPL126W & AFUA_7G02610 & -0.101632712 \\
\hline YDL189W & AFUA_1G09400 & -0.104331651 \\
\hline YHL016C & AFUA_1G04870 & -0.106608293 \\
\hline YLR380W & AFUA_4G13930 & -0.111437921 \\
\hline YPR165W & AFUA_6G06900 & -0.117573203 \\
\hline YOL062C & AFUA_5G07930 & -0.122120003 \\
\hline YGL248W & AFUA_1G14890 & -0.124318908 \\
\hline YLR029C & AFUA_1G04660 & -0.129375946 \\
\hline YMR214W & AFUA_2G08300 & -0.130867913 \\
\hline YOR124C & AFUA_6G12270 & -0.136050424 \\
\hline YOL023W & AFUA_1G06520 & -0.136914472 \\
\hline YPL280W & AFUA_3G01210 & -0.13706189 \\
\hline YLR380W & AFUA_7G06760 & -0.141209958 \\
\hline
\end{tabular}


Table 4: Continued.

\begin{tabular}{|c|c|c|}
\hline S. cerevisiae gene & A. fumigatus gene & Correlation coefficient \\
\hline YNL241C & AFUA_3G08470 & -0.153108798 \\
\hline YOR391C & AFUA_3G01210 & -0.154014157 \\
\hline YOL089C & AFUA_6G02330 & -0.161608796 \\
\hline YGL215W & AFUA_3G10040 & -0.170171082 \\
\hline YMR137C & AFUA_2G15220 & -0.174259735 \\
\hline YER004W & AFUA_6G08930 & -0.179730339 \\
\hline YDR365C & AFUA_2G05420 & -0.181946191 \\
\hline YDR529C & AFUA_4G06790 & -0.185927497 \\
\hline YDR062W & AFUA_6G00300 & -0.189190157 \\
\hline YOL086C & AFUA_7G01010 & -0.190813468 \\
\hline YOL026C & AFUA_5G03630 & -0.19138562 \\
\hline YIL033C & AFUA_3G10000 & -0.195925157 \\
\hline YBR290W & AFUA_4G13740 & -0.19955841 \\
\hline YAR019C & AFUA_4G06750 & -0.204744755 \\
\hline YHL007C & AFUA_2G04680 & -0.20737573 \\
\hline YMR078C & AFUA_7G05480 & -0.222707833 \\
\hline YDR311W & AFUA_4G11690 & -0.22686955 \\
\hline YOL100W & AFUA_3G12670 & -0.230714484 \\
\hline YGR162W & AFUA_2G09490 & -0.232235851 \\
\hline YGL255W & AFUA_1G01550 & -0.248116851 \\
\hline YPL038W & AFUA_6G01910 & -0.252388553 \\
\hline YCL035C & AFUA_1G06100 & -0.255759517 \\
\hline YNL082W & AFUA_2G13410 & -0.259394819 \\
\hline YDR062W & AFUA_1G11890 & -0.263523207 \\
\hline YOR390W & AFUA_2G16210 & -0.269616243 \\
\hline YJR141W & AFUA_3G07970 & -0.2738444 \\
\hline YOR365C & AFUA_2G17650 & -0.274036262 \\
\hline YDL116W & AFUA_1G10860 & -0.278315121 \\
\hline YKL020C & AFUA_1G12550 & -0.278379939 \\
\hline$Y D L 174 C$ & AFUA_3G06820 & -0.286419799 \\
\hline YBR029C & AFUA_1G07010 & -0.296926336 \\
\hline YLR096W & AFUA_1G11080 & -0.297540349 \\
\hline YFL028C & AFUA_6G05080 & -0.303502181 \\
\hline YOR089C & AFUA_3G10740 & -0.30892361 \\
\hline YPR010C & AFUA_2G13000 & -0.309890067 \\
\hline YJL219W & AFUA_5G01160 & -0.321415181 \\
\hline YNL097C & AFUA_4G11660 & -0.329160424 \\
\hline$Y M R 116 C$ & AFUA_4G13170 & -0.329415498 \\
\hline YPL280W & AFUA_5G01430 & -0.331554435 \\
\hline YHR091C & AFUA_2G14030 & -0.337931682 \\
\hline YIL030C & AFUA_2G08650 & -0.350942099 \\
\hline YFR037C & AFUA_7G05510 & -0.356342064 \\
\hline YOR391C & AFUA_5G01430 & -0.356843862 \\
\hline$Y D L 174 C$ & AFUA_1G00510 & -0.402217656 \\
\hline$Y M L 121 W$ & AFUA_5G09650 & -0.410825283 \\
\hline YFL060C & AFUA_2G08580 & -0.411505108 \\
\hline YDR301W & AFUA_8G04040 & -0.413112754 \\
\hline YJL221C & AFUA_3G07380 & -0.413321446 \\
\hline YLR326W & AFUA_5G12410 & -0.458648708 \\
\hline
\end{tabular}


TABle 4: Continued.

\begin{tabular}{lcr}
\hline S. cerevisiae gene & A. fumigatus gene & Correlation coefficient \\
\hline YBL108C-A & AFUA_4G03360 & -0.487139446 \\
$Y G R 165 W$ & AFUA_5G08380 & -0.519339895 \\
$Y K L 157 W$ & AFUA_4G09030 & -0.604021491 \\
$Y N L 334 C$ & AFUA_2G08580 & -0.720610634 \\
\hline
\end{tabular}

Nucleosome of YIR038C

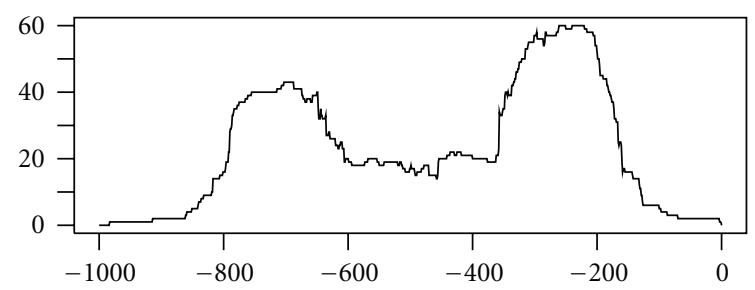

(a)

Nucleosome of AFUA_8G02500

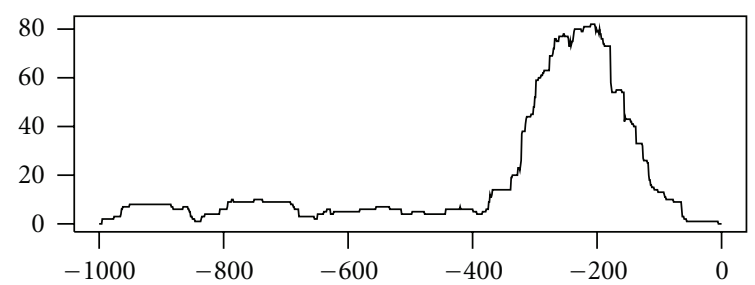

(c)

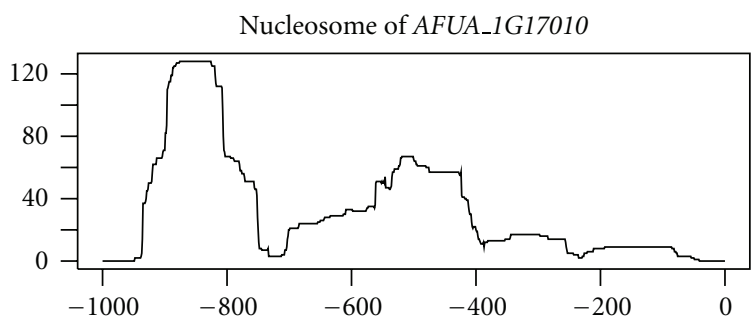

(e)

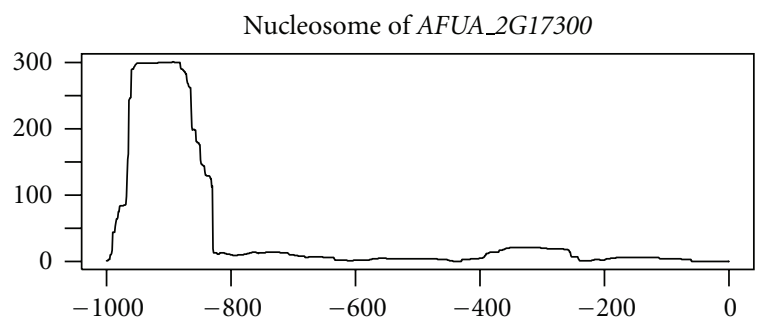

(g)
TSS of YIR038C

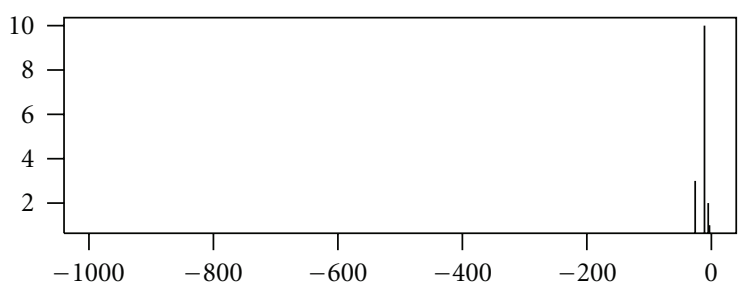

(b)

TSS of AFUA_00520G8

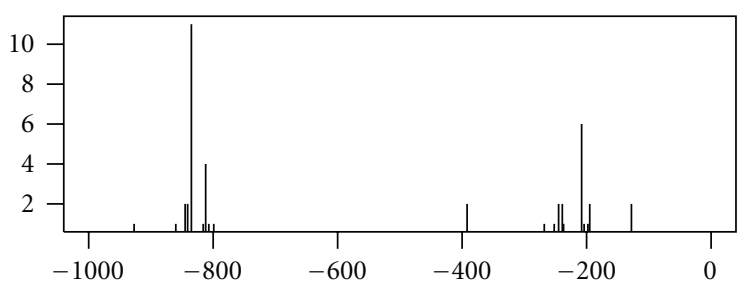

(d)

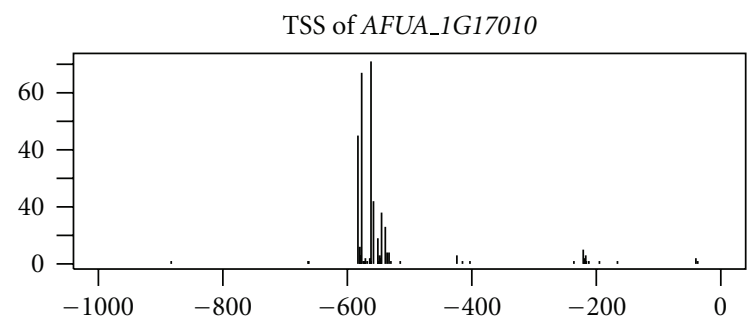

(f)

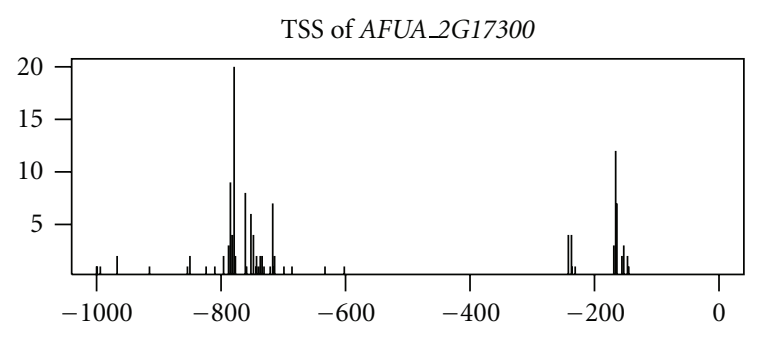

(h)

FIgURE 3: Mapping numbers of nucleosomes and transcription start sites in the promoter regions of YIR038C, AFUA_8G02500, AFUA_1G17010, and AFUA_2G17300. Position 0 indicates the translational start site.

Single-gene duplications and gene cluster duplications consisting of multiple genes were identified. One cluster of 4 genes (AFUA_1G00420 to AFUA_1G00470) is a duplication of another 4-gene cluster (AFUA_8G04120 to AFUA_8G04080) (Table 2). Among these gene pairs, the nucleosome position profile was poorly conserved in the gene promoter between
AFUA_1G00470 and AFUA_8G04080 and in the gene body between AFUA_1G00440 and AFUA_8G04110 (Spearman's rank correlation coefficients were 0.43 and 0.23 , resp.) (Table 2). With the exception of these 2 cases, the nucleosome position profile was highly conserved (correlation coefficients were higher than 0.7) (Table 2). 
We analyzed another pair of duplicated clusters ( 9 genes) (AFUA_1G16030 to AFUA_1G16120 and AFUA_5G14930 to AFUA_5G15030). The genes in each cluster have evolved for the same period after the duplication (Table 2). At present, conservation of the nucleosome position profiles varies among the 9 genes (Table 2). For example, the nucleosome position profile is poorly conserved in the gene promoters of 3 gene pairs (AFUA_1G16050 and AFUA_5G14950, AFUA_16110 and AFUA_15020, AFUA_1G16120 and AFUA_15030) (Spearman's rank correlation coefficients are $-0.35,-0.26$, and -0.14 , resp.). On the other hand, the nucleosome position profile is highly conserved in the promoters of AFUA_16070 and AFUA_5G14980 and was strongly correlated (correlation coefficient $=0.93$ ). These results suggest that transcriptional regulation of duplicated genes is associated with nucleosome positions in the gene promoters.

\subsection{Nucleosome Position Profiles of Orthologous Gene Promot-} ers in Aspergillus fumigatus and Saccharomyces cerevisiae. I compared the nucleosome position profiles in the promoters of 347 orthologous pairs of yeast genes that showed notably high conservation in the control and mutant strains. In the 63 duplicated A. fumigatus gene pairs, $13(20.6 \%)$ gene promoter profiles and $11(17.5 \%)$ gene body profiles were highly correlated (Spearman's rank correlation coefficient $>0.7$ ) (Table 1, Figure 1). On the other hand, of the 347 orthologous gene pairs, only 11 (3.2\%) nucleosome position profiles were highly correlated (Spearman's rank correlation coefficient $>0.7$ ) (Table 4, Figure 2). The distribution of correlation coefficients of the 347 orthologous gene promoters did not significantly differ from that of the control (gene pairs chosen at random $)(P$-value $=0.28$ Kolmogorov-Smirnov test). One potential cause of this low conservation is the large evolutionary distance between the 2 fungi. A. fumigatus and S. cerevisiae belong to the subphyla Pezizomycotina and Saccharomycotina, respectively. Alternatively, this low conservation may represent a difference in mechanisms regulating the nucleosome arrangement, since the nucleosomal (nucleosome-bound) DNA lengths differ between the 2 fungi $[9,10]$.

Nucleosome position profiles in gene promoters are thought to be related to gene function. For example, YIR038C of S. cerevisiae encodes an amino acid sequence protein (glutathione S-transferase) similar to 3 genes (AFUA_1G17010, AFUA_2G17300, and AFUA_8G02500) in A. fumigatus (Table 4). Although the nucleosome position profiles show some conservation between YIR038C and AFUA_8G02500 (Spearman's rank correlation coefficient $=0.55$, except for one nucleosome position loss in $A$. fumigatus), they are poorly conserved between YIR038C and AFUA_1G17010 (Spearman's rank correlation coefficient $=-0.03$ ) and between YIR038C and AFUA_2G17300 (Spearman's rank correlation coefficient $=-0.07)($ Table 4 , Figure 3).

Interestingly, although the nucleosome position profile of AFUA_8G02500 is completely different from that of AFUA_2G17300, the transcription start site patterns are very similar between these genes (Figure 3), suggesting that the relationship between transcription start site and nucleosome position in the gene promoter varies.

\section{Acknowledgments}

The author thanks Dr. Shinji Kondo for helpful comments and critical review of the paper. This study was supported in part by a grant from the Institute for Fermentation, Osaka (IFO), Japan.

\section{References}

[1] K. Luger, A. W. Mäder, R. K. Richmond, D. F. Sargent, and T. J. Richmond, "Crystal structure of the nucleosome core particle at $2.8 \AA$ resolution,” Nature, vol. 389, no. 6648, pp. 251-260, 1997.

[2] C. K. Lee, Y. Shibata, B. Rao, B. D. Strahl, and J. D. Lieb, "Evidence for nucleosome depletion at active regulatory regions genome-wide," Nature Genetics, vol. 36, no. 8, pp. 900905, 2004.

[3] G. C. Yuan, Y. J. Liu, M. F. Dion et al., "Molecular biology: genome-scale identification of nucleosome positions in $S$. cerevisiae," Science, vol. 309, no. 5734, pp. 626-630, 2005.

[4] H. Nishida, T. Motoyama, Y. Suzuki, S. Yamamoto, H. Aburatani, and H. Osada, "Genome-wide maps of mononucleosomes and dinucleosomes containing hyperacetylated histOnes of Aspergillus fumigatus," PloS One, vol. 5, no. 3, article e9916, 2010.

[5] A. M. Tsankov, D. A. Thompson, A. Socha, A. Regev, and O. J. Rando, "The role of nucleosome positioning in the evolution of gene regulation," PLoS Biology, vol. 8, no. 7, article e1000414, 2010

[6] E. Segal, Y. Fondufe-Mittendorf, L. Chen et al., "A genomic code for nucleosome positioning," Nature, vol. 442, no. 7104, pp. 772-778, 2006.

[7] A. B. Lantermann, T. Straub, A. Strålfors, G. C. Yuan, K. Ekwall, and P. Korber, "Schizosaccharomyces pombe genomewide nucleosome mapping reveals positioning mechanisms distinct from those of Saccharomyces cerevisiae," Nature Structural and Molecular Biology, vol. 17, no. 2, pp. 251-257, 2010.

[8] S. A. Teichmann and M. M. Babu, "Gene regulatory network growth by duplication," Nature Genetics, vol. 36, no. 5, pp. 492-496, 2004.

[9] H. Nishida, T. Motoyama, S. Yamamoto, H. Aburatani, and H. Osada, "Genome-wide maps of mono- and di-nucleosomes of Aspergillus fumigatus," Bioinformatics, vol. 25, no. 18, pp. 2295-2297, 2009.

[10] T. Matsumoto, C.-S. Yun, H. Yoshikawa, and H. Nishida, "Comparative studies of genome-wide maps of nucleosomes between deletion mutants of elp3 and hos 2 genes of Saccharomyces cerevisiae," PLoS One, vol. 6, no. 1, article e16372, 2011.

[11] H. Nishida, "Evolutionary conservation levels of subunits of histOne-modifying protein complexes in fungi," Comparative and Functional Genomics, vol. 2009, Article ID 379317, 6 pages, 2009.

[12] I. Uchiyama, T. Higuchi, and M. Kawai, "MBGD update 2010: toward a comprehensive resource for exploring microbial genome diversity," Nucleic Acids Research, vol. 38, no. 1, pp. D361-D365, 2009.

[13] H. Nishida, "Calculation of the ratio of the mononucleosome mapping number to the dinucleosome mapping number for 
each nucleotide position in the Aspergillus fumigatus genome," Open Access Bioinformatics, vol. 1, pp. 1-6, 2009.

[14] D. E. SchOnes, K. Cui, S. Cuddapah et al., "Dynamic regulation of nucleosome positioning in the human genome," Cell, vol. 132, no. 5, pp. 887-898, 2008. 

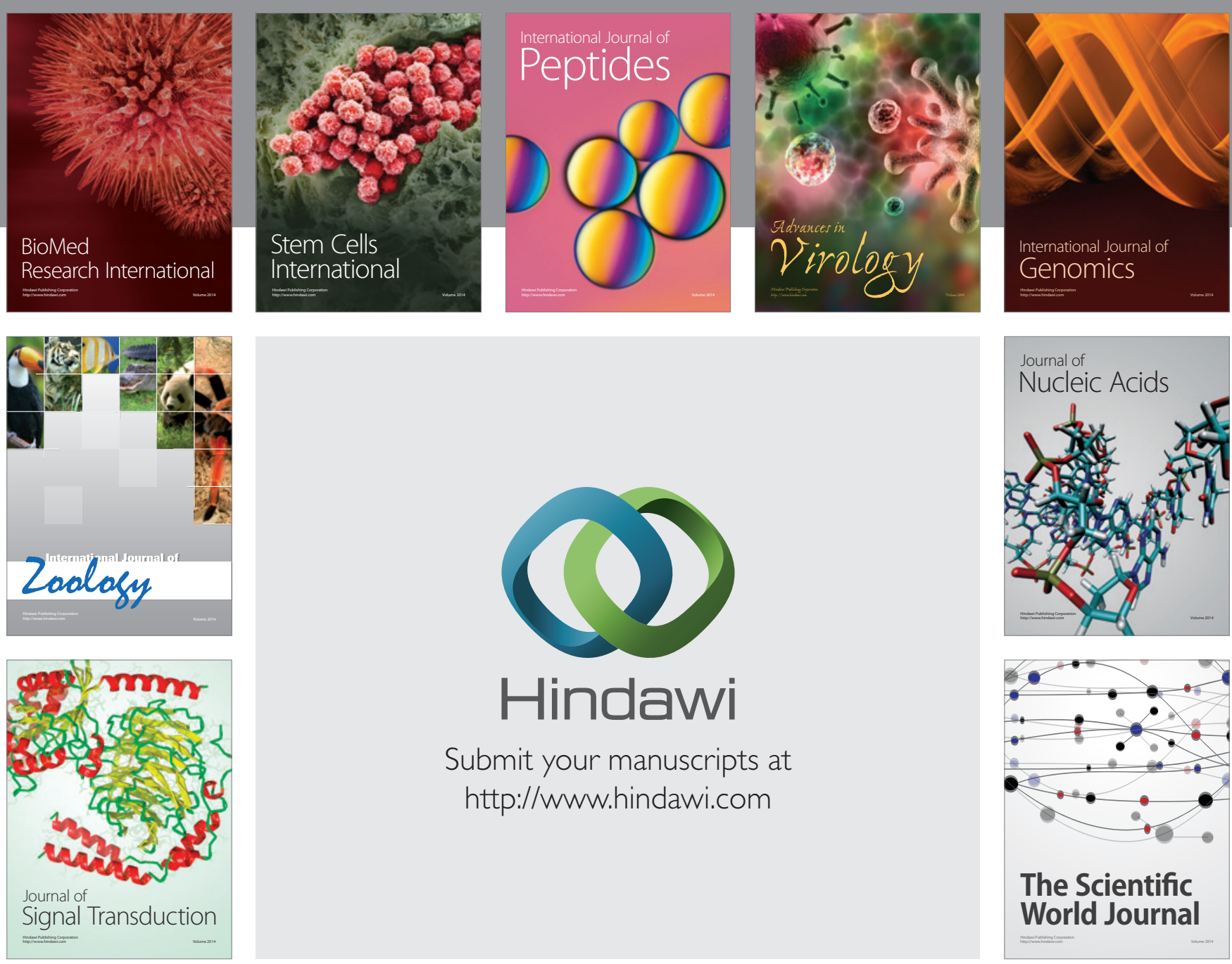

Submit your manuscripts at

http://www.hindawi.com
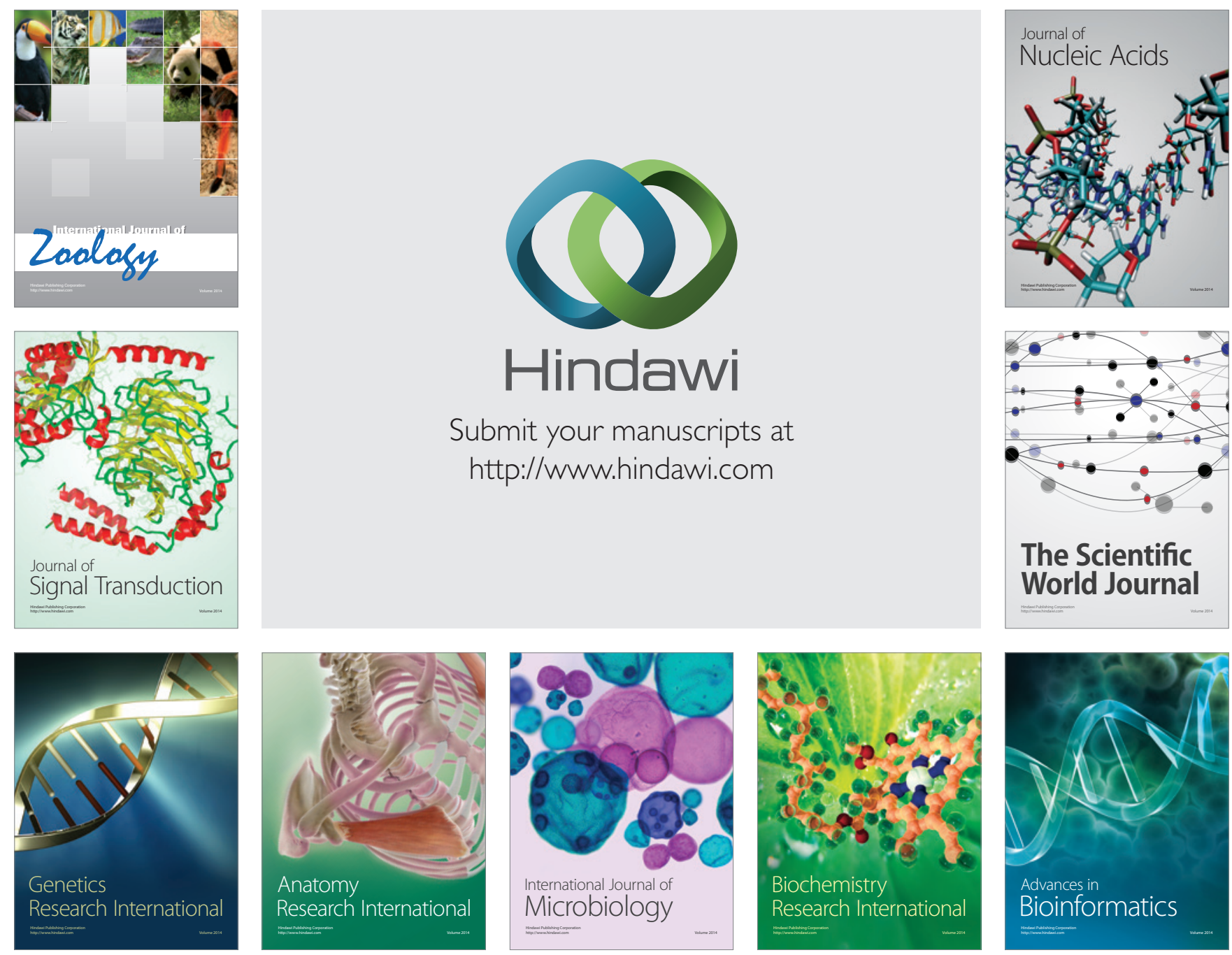

The Scientific World Journal
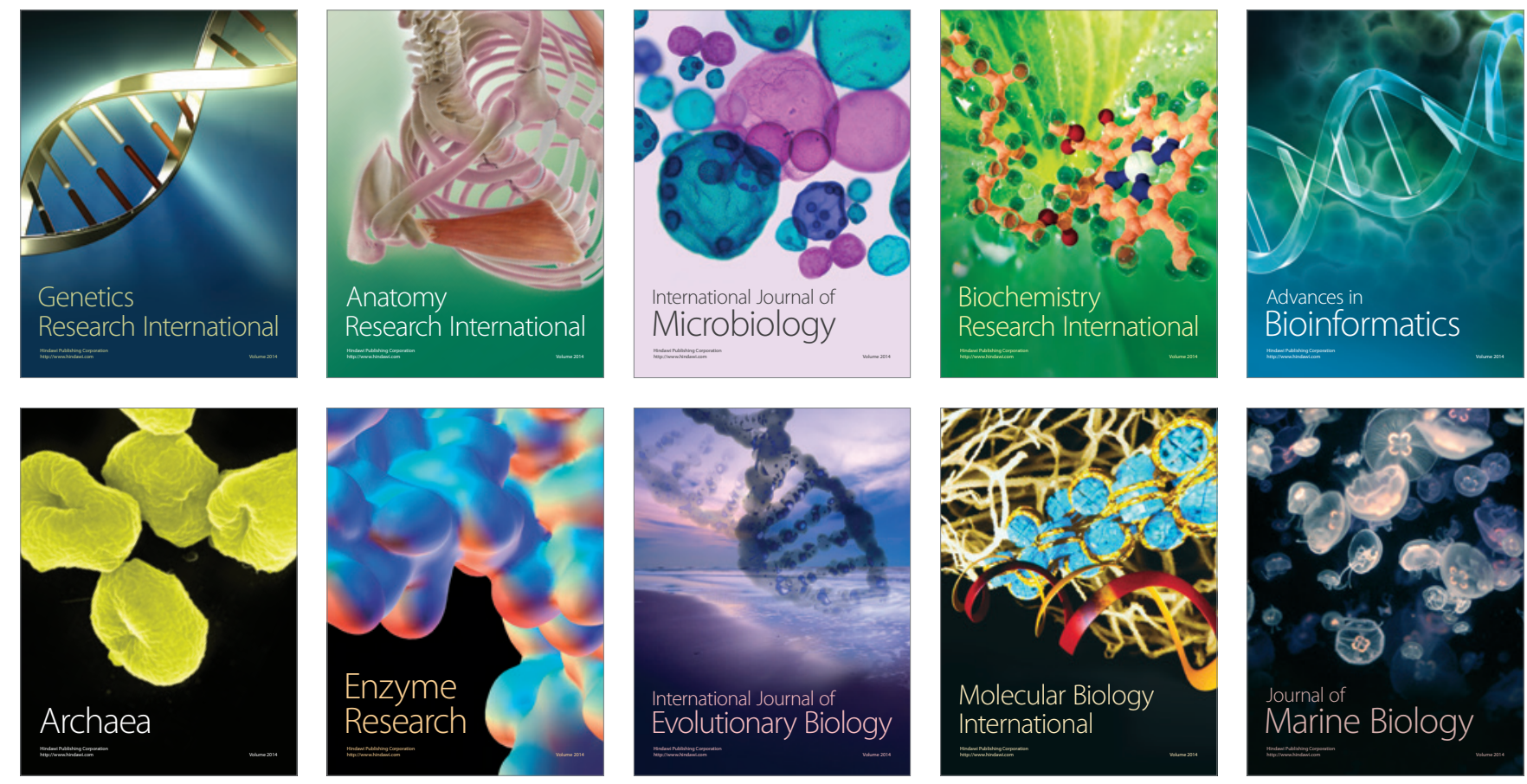